\title{
Fluctuations, response and aging dynamics in a simple glass-forming liquid out of equilibrium.
}

\author{
Walter Kob \\ Institut für Physik, Johannes Gutenberg-Universität, Staudinger Weg 7, D-55099 Mainz, Germany \\ Jean-Louis Barrat \\ Département de Physique des Matériaux \\ Université Claude Bernard and CNRS, 69622 Villeurbanne Cedex, France
}

\begin{abstract}
By means of molecular dynamics computer simulations we investigate the out of equilibrium relaxation dynamics of a simple glass former, a binary Lennard-Jones system, after a quench to low temperatures. We find that one-time quantities, such as the energy or the structure factor, show only a weak time dependence. By comparing the out of equilibrium structure factor with equilibrium data we find evidence that during the aging process the system remains in that part of phase space that mode-coupling theory classifies as liquid like. Two-times correlation functions show a strong time and waiting time $t_{w}$ dependence. For large $t_{w}$ and times corresponding to the early $\beta$-relaxation regime the correlators approach the Edwards-Anderson value by means of a power-law in time. For large but fixed values of $t_{w}$ the relaxation dynamics in the $\beta$-relaxation regime seems to be independent of the observable and temperature. The $\alpha$-relaxation shows a power-law dependence on time with an exponent which is independent of $t_{w}$ but depends on the observable. We find that at long times $\tau$ the correlation functions can be expressed as $C_{\mathrm{AG}}\left(h\left(t_{w}+\tau\right) / h\left(t_{w}\right)\right)$ and compute the function $h(t)$. This function is found to show a $t$-dependence which is a bit stronger than a logarithm and to depend on the observable considered. If the system is quenched to very low temperatures the relaxation dynamics at long times shows fast drops as a function of time. We relate these drops to relatively local rearangements in which part of the sample relaxes its stress by a collective motion of 50-100 particles. Finally we discuss our measurements of the time dependent response function. We find that at long times the correlation functions and the response are not related by the usual fluctuation dissipation theorem but that this relation is similar to the one found for spin glasses with one step replica symmetry breaking.
\end{abstract}

PACS numbers: 61.20.Lc, 61.20.Ja, 02.70.Ns, 64.70.Pf

\section{INTRODUCTION}

It is well known from experiments that the most prominent feature of supercooled liquids and glass forming systems is the rapid increase of their relaxation time as temperature is decreased [1]. Since the early eighties, computer simulations have become a powerful tool to investigate such systems and hence to increase our understanding of their extraordinary dynamical behavior [2]. However, because of the rapid increase of the relaxation times these simulations are faced with the intrinsic problem that, over a large range of temperature, systems cannot be equilibrated, as the simulation time, typically $10^{-7} \mathrm{~s}$ for an atomic system, is smaller than the relaxation time. This means that on a computer, any system with a longer relaxation time can only be studied under nonequilibrium conditions. In other words, in computer simulations the "glass transition" always takes place at temperatures higher than in real experiments.

Despite this drawback, it has been shown that computer simulations can provide useful information on the mechanisms than underly the rapid increase of the time scales in supercooled liquids. One of the most valuable contributions in this direction was the proof that the so called mode-coupling theory [3., 1 . does indeed provide a quantitative description of this slowing down within the time window explored in the simulation, at least for those simple atomic liquids that are "fragile glass formers", i.e. show a strongly non-Arrhenius dependence of the relaxation times as a function of temperature. The findings from computer simulations [2] and experiments [4] have largely confirmed that this theory provides a consistent picture for the dynamical behaviour of such liquids over a relaxation time range that covers several decades, typically $10^{-11}-10^{-8} \mathrm{~S}$.

For larger relaxation times (lower temperatures), equilibrium simulations are no longer possible. On the other hand, one can take advantage of the flexibility of the simulation to explore the nonequilibrium properties on times scales that would be rather difficult to access in experiments. This possibility stems from the fact that the simulations allows instantaneous quenches to low temperatures, and a monitoring of the subsequent evolution on a fast time scale. Experimentally, the study of nonequilibrium evolution in glassy systems following a fast quench is a field that has been explored since a long time in the so called "aging" experiments [5], performed on a time scale of hours or even days. 
More specifically, "physical aging" consists in a slow evolution of the characteristic properties of glassy systems that do not undergo any changes in their chemical composition. Of particular interest is the evolution of response functions (typically the elastic compliance) that are usually found, e.g. in polymeric systems, to exhibit a strong slowing down as the waiting time (i.e. the time elapsed since the quench) increases. The recent interest of the physics community in this behavior was aroused when a number of experiments performed on spin glasses showed that a very similar behavior could be observed in these disordered magnetic systems. In particular it was found that the response to a magnetic field of a system that has been quenched into its low temperature, nonequilibrium spin glass phase, becomes more and more sluggish as the waiting time increases [6]. Subsequently, a number of theories were put forward to explain this aging behavior of spin glass systems from first principles statistical physics. A comprehensive review of these theories can be found in reference [7]. Essentially, one is lead to distinguish between phenomenological "trap models", domain growth theories, and mode-coupling or mean field theories. "Trap models" describe the evolution of systems as a random walk in a complex phase space, which can under certain conditions give rise to aging. The domain growth models assume that the aging results from a coarsening process somewhat similar to what can be observed in a ferromagnetic system quenched below its critical temperature. Mean field or mode-coupling theories account exactly for the aging behaviour of some disordered models in the limit of high dimension (e.g. a particle in a random potential in a high dimensional space). Interestingly, these mean field theories give rise to a mathematical structure that is very similar to that of the mode-coupling theory commonly used to describe the dynamical properties of supercooled liquids [3, 1]. A major difference, however, is that high dimensional systems can be shown to have a true transition towards a non-ergodic behavior as the temperature is lowered, whereas it is well known that the transition predicted by the mode-coupling theory of liquids is smeared out and does not give rise to a true singularity. If, however, a true transition is present, a natural consequence is that a system quenched below the transition temperature will display aging behavior. Theoretically, this aging behavior of mean field systems was studied in great detail and is now quite well understood [7]. The question arises, however, to what extent this description is relevant to the aging behaviour of real, three dimensional systems. In particular, in view of the mathematical similarity mentioned above, it is quite natural to inquire whether the nonequilibrium dynamics of those supercooled liquids that are known to be well described by mode-coupling theory can be accounted for by the mean field description of aging.

In an attempt to partially answer this question, we present molecular dynamics simulations of a simple glass forming liquid, whose properties under equilibrium conditions have been extensively studied in previous work [8]. We will concentrate on the characterization of the "aging" behaviour of such a system on the time scales that can be investigated in molecular dynamics simulations, i.e. a few nanoseconds. Once again, we emphasize that these time scales are very different from those usually investigated in aging experiments on glasses (although some recent dielectric spectroscopy experiments [9] 10] investigate relatively high frequency behaviour). Moreover, the systems under study would, from an experimental viewpoint, be considered as supercooled liquids rather than glasses. Glassy behavior is observed only on the restricted time scale of the computer simulation. The possibility of exploring such relatively short time scales is nevertheless interesting, as it is precisely on such time scales that mode-coupling theory is successful. Moreover, the simulation offers the opportunity to simultaneously compute time dependent correlation functions, static quantities and response functions. Relating these quantities to each other is an essential achievement in mean field theories of aging behaviour, whose predictions can then be tested in detail.

The paper is organized as follows. In section II, we recall the main features of our model and describe some technical details of the simulations. Sections III and IV describe the aging behavior of static properties and of correlation functions, respectively. Section $\mathrm{V}$ deals with the aging behaviour of response functions. Preliminary accounts of these results can be found in [11]. Some closely related studies, on a slightly different system, have been reported in [12].

\section{MODEL AND DETAILS OF THE SIMULATION}

The model we use is a binary (80:20) mixture of particles, which in the following we will call A and B particles, that interact via a Lennard-Jones potential, $V_{\alpha \beta}(r)=4 \epsilon_{\alpha \beta}\left\{\left(\sigma_{\alpha \beta} / r\right)^{12}-\left(\sigma_{\alpha \beta} / r\right)^{6}\right\}$, with $\alpha, \beta \in\{\mathrm{A}, \mathrm{B}\}$. The constants $\epsilon_{\alpha \beta}$ and $\sigma_{\alpha \beta}$ are given by $\epsilon_{\mathrm{AA}}=1.0, \sigma_{\mathrm{AA}}=1.0, \epsilon_{\mathrm{AB}}=1.5, \sigma_{\mathrm{AB}}=0.8, \epsilon_{\mathrm{BB}}=0.5$, and $\sigma_{\mathrm{BB}}=0.88$ and the potential is cut off and shifted at a distance $2.5 \sigma_{\alpha \beta}$. In the following we will report the results in reduced units, with $\sigma_{\mathrm{AA}}$ and $\epsilon_{\mathrm{AA}}$ the unit of length and energy, respectively (setting the Boltzmann constant $k_{B}=1.0$ ). Time will be measured in units of $\sqrt{\sigma_{\mathrm{AA}}^{2} m / 48 \epsilon_{\mathrm{AA}}}$, where $m$ is the mass of the particles.

The simulation was done with $N=1000$ particles and at constant volume $V=L^{3}$, with a box length $L=9.4$. At this density the equilibrium dynamics of the system has been investigated intensively [8, 13] and it has been found that at low temperatures, $0.446 \leq T \leq 0.8$, this dynamics is described very well by mode-coupling theory [3,4] with a critical temperature $T_{c}=0.435$. Although there is evidence that for temperatures $T \leq 0.452$ the increase of 
the relaxation times, or the inverse of the diffusion constant, is less strong than the power-law predicted by MCT, $\left(T-T_{c}\right)^{-\gamma}$, with $\gamma=2.3$ [14], the increase is still so strong that at temperatures less than $T_{c}$ the relaxation time of the system exceeds by orders of magnitude the time scale accessible to a present state of the art computer simulation $\left(O\left(10^{7}\right)\right.$ time units). Hence for a computer simulation $T_{c}$ plays the role of the glass transition temperature $T_{g}$.

The equations of motion were integrated with the velocity form of the Verlet algorithm, with a step size of 0.02 . The system was equilibrated in the high temperature phase at a temperature $T_{i}>T_{c}$ and then quenched at time $t=0$ to a temperature $T_{f} \leq T_{c}$. This quench was done by means of a stochastic heat bath, i.e. every 50 time steps we replaced the velocities of all the particles with ones that were drawn from a Maxwell-Boltzmann distribution corresponding to a temperature $T_{f}$. In order to study the dependence of the relaxation behavior on the initial and final temperature we considered several values of $T_{i}$ and $T_{f}: T_{i} \in\{5.0,0.8,0.466\}$ and $T_{f} \in\{0.435,0.4,0.3,0.1\}$.

After the quench we propagated the system for a waiting time $t_{w}$ after which the measurements of the quantities of interest were started. In the course of the simulations we realized that there are appreciable sample to sample variations of the relaxation curves. Therefore it was necessary to average for each combination of $T_{i}$ and $T_{f}$ over 8-10 independent runs.

\section{ONE-TIME QUANTITIES}

One-time quantities are observables which in equilibrium are constants, such as the total energy of the system, the pressure or, in a magnetic system, the magnetization. In the non-equilibrium situation these observables depend on the time which has elapsed since the quench and thus are commonly called "one-time" quantities.

It has been shown before, see e.g. Refs. [11, 15, 12], that the time dependence of such quantities is relatively weak. In Fig. 1 we show the time dependence of $e_{\text {pot }}(t)$, the potential energy per particle, for various combinations of $T_{i}$ and $T_{f}$. From the figure it becomes evident that this time dependence is rather weak. In Ref. 11] we have shown that it can be approximated well by a power-law with a small exponent (0.14), or alternatively by a logarithmic time dependence. Qualitatively this result agrees with the one of Monte Carlo simulations of a soft sphere system [12] and a polymer model [15] in which also power-law dependences have been observed for one-time quantities. However, the exponents found in these simulations $(0.7$ and $1 / 3)$ are significantly larger than our, thus indicating that they are not universal quantities. The large exponent of reference [12] might, however, be also due to the fact that in that simulation $T_{i}$ was infinity and that the runs were rather short, i.e. that the dependence of $e_{\text {pot }}$ on time was not the one valid for very long times.

From Fig. 1 1 a we conclude that at long times the curves do not depend significantly on the value of the initial temperature (see the curves with $T_{f}=0.4$ ). For short times, however, such a dependence can be seen, in that, e.g., the curve with $T_{i}=0.466$ is almost constant and shows only at long times a time dependence which is similar to the one for higher values of $T_{i}$ (see also Ref. [11]). This time dependence starts to be observable only for times that are comparable with the $\alpha$-relaxation time of the system at $T_{i}$, which for $T_{i}=0.466$ is on the order of $10^{4}$ time units [8], since this is the typical time scale that the system needs to find a significantly different configuration. This estimate is, however, only a lower bound, since the dynamics of the system is given by the temperature $T_{f}<T_{i}$, and is hence slower than the one at $T_{i}$. See also Ref. [16] for a further discussion of this point.

The figure also shows that there is a significant dependence of the curves on $T_{f}$ in that the values of $e_{\text {pot }}(t)$ decrease with decreasing $T_{f}$. In a harmonic solid at a temperature $T_{f}$ one expects that the (equilibrium!) potential energy varies like $3 / 2 k_{B} T_{f}$ and thus it is reasonable to subtract this "trivial contribution" from the curves. The result of this subtraction is shown in Fig. $1 \mathrm{~b}$ from which we see that this procedure does indeed make the curves collapse reasonably well. The most significant exceptions are the curves $\left(T_{i}=0.466, T_{f}=0.4\right)$ and $\left(T_{i}=5.0, T_{f}=0.1\right)$. These deviations can be understood by realizing that during the aging process the system is looking for configurations with low lying energies. If this search is started at a relatively low temperature, such as $T_{i}=0.466$, the system will typically be able to find configurations that have a lower potential energy than in the case when $T_{i}$ is large thus explaining why the curve for $T_{i}=0.466$ is below the other ones. In addition to this, such a search will be more efficient at a higher temperature $T_{f}$ than at a low one, since then the system has a better chance to overcome small local barriers. This explains why the curve for $\left(T_{i}=5.0, T_{f}=0.1\right)$ is above the other ones with the same value of $T_{i}$.

Since also the radial distribution function $g(r)$ is a one-time quantity we expect that also its dependence on time is weak. That this is indeed the case is shown in the main part of Fig. 2 where we show $g_{\mathrm{AA}}(r)$, i.e. the partial radial distribution function for the A particles (see Ref. [17] for its definition) for the times $t=0$ (i.e. before the quench), $t=10,100,1000,10000$, and 63100 time units. From the figure we see that immediately after the quench $g_{\mathrm{AA}}(r)$ changes its shape very quickly in that, e.g., the first nearest neighbor peak which in the high temperature phase (curve labeled with $t=0$ ) is not very high, becomes much higher and narrower. For times larger than 10, the shape becomes essentially independent of time, as expected for a one-time quantity and in agreement with the results of Ref. [12]. 
That the form of $g_{\mathrm{AA}}(r)$ at long times has a significant dependence on $T_{f}$ is demonstrated in the inset of Fig. 2, where we show the main peak for different values of $T_{f}$ at long times $(t=63100)$. As expected we find that the height of this peak increases with decreasing temperature and that it becomes narrower. Thus we conclude that the typical configurations in which the system is stuck after long times does depend on the final temperature. Very recently Latz made the interesting proposition that the typical configurations in which the system is stuck at long times during the aging process share a common property in that all of them are very close to the so-called critical surface of mode-coupling theory (MCT) [3 3 ] which divides the liquid like phase of the system from its glass like phase [18]. MCT predicts that this critical surface can be calculated by using as input the radial distribution function, i.e. a purely static quantity [3, An. Furthermore it has been shown that, for simple liquids, the relevant part of this input is related to the area under the first peak in the structure factor 3, 39], or alternatively, to the area under the first peak in $g(r)$ weighted with $4 \pi r^{2}$. In order to test the validity of the proposition by Latz we have therefore calculated the integral of $4 \pi r^{2} g_{\mathrm{AA}}(r)$ between zero and 1.406, the location of the first minimum in $g_{\mathrm{AA}}(r)$. Note that this integral, which we call $c$, is the partial coordination number. The time and $T_{f}$ dependence of $c$ are shown in the main part of Fig. 3 (open symbols). (In order to expand the axis at low temperatures we show this data versus the logarithm of $T_{f}$.). The times $t$ shown are spaced essentially equidistant on a logarithmic time axis and are $0,10,40,60,100$, $160,250,400,630,1000,1580,2510,3980,6310,10000,15850,25120,39810$, and 63100 . (In order to show the time dependence we plot the data for $t=63100$ at $T=T_{f}$ and with decreasing $t$ at a temperature which is a factor of 1.003 higher than the previous data point.) From this figure we recognize that with increasing time the value of $c$ increases rapidly and then becomes constant to within the precision of our data. The value of this limiting constant, which we call $c_{\infty}$, increases weakly with increasing $T_{f}$ but to a first approximation it can be considered as independent of $T_{f}$, thus supporting the prediction of Latz.

In order to see whether the $T_{f}$ dependence of $c_{\infty}$ is indeed weak it is useful to compare it with the temperature dependence of the area under the first peak $g_{\mathrm{AA}}$ in equilibrium. For this we have included in the figure also the value of $c$ for temperatures $5.0 \geq T \geq 0.446$ (filled symbols). (The $g_{\mathrm{AA}}(r)$ for the various temperatures are results from other simulations [8,13].) In this temperature range the equilibrium value of $c$ shows an appreciable temperatures dependence (which is close to a logarithmic dependence) which is indeed much more pronounced than the $T_{f}$ dependence of $c_{\infty}$ of the non-equilibrium simulations. To a first approximation the value of $c_{\infty}$ is given by the value of $c$ of the equilibrium curve at $T=T_{c}$, where $T_{c}$ is the so-called critical temperature of MCT 18]. For the present system this critical temperature has been estimated to be around $T=0.435$ [8,20]. Due to the problem to equilibrate the system at temperatures close to $T_{c}$ it is currently not possible to determine the equilibrium value of $c$ at $T_{c}$ and therefore to compare this value with the $c_{\infty}$ from the out-of-equilibrium simulations. However, since the equilibrium value of $c$ is expected to show a smooth temperature dependence, it can be estimated quite reliably from the equilibrium data at a bit higher temperatures. In the inset of Fig. 3 we show an enlargement of the region which is marked by a box in the main figure and which encloses the temperature range around $T_{c}$. From this inset we see that the values of $c_{\infty}$ for $T_{f}=T_{c}=0.435$ and $T_{f}=0.4$ are indeed very close to an extrapolation of the equilibrium curve to $T_{c}$. Hence we conclude that it is indeed possible to calculate from the equilibrium data with reasonable accuracy also certain quantities for the out-of-equilibrium situation. This point is discussed in more detail in Ref. [16].

\section{TWO-TIMES QUANTITIES}

\section{A. General features of two time correlation functions}

In equilibrium, time correlation functions between any two observables $A$ and $B,\langle A(\tau) B(0)\rangle$ depend only on the time difference $\tau$, i.e. $\langle A(\tau) B(0)\rangle=\left\langle A\left(\tau+t_{w}\right) B\left(t_{w}\right)\right\rangle$, for all waiting times $t_{w}$. For the out of equilibrium situation this equality no longer holds and therefore such time correlation functions depend on two quantities, the time difference $\tau$ and $t_{w}$, the time passed since the generation of the non-equilibrium situation. Therefore such correlation functions are called two-times quantities and in this subsection we will demonstrate that such quantities are very well suited to investigate the aging dynamics of out of equilibrium systems.

For liquids in equilibrium the dynamics is often studied by means of the intermediate scattering function $F(k, t)$ which is defined by [17]

$$
F(k, \tau)=\frac{1}{N} \sum_{j, l} \exp \left(i \mathbf{k} \cdot\left(\mathbf{r}_{j}(\tau)-\mathbf{r}_{l}(0)\right)\right.
$$

and by the its so-called self part, $F_{s}(k, t)$ given by 


$$
F_{s}(k, \tau)=\frac{1}{N} \sum_{j} \exp \left(i \mathbf{k} \cdot\left(\mathbf{r}_{j}(\tau)-\mathbf{r}_{j}(0)\right)\right.
$$

Here $\mathbf{r}_{k}(\tau)$ are the positions of the particles at time $t$ and $\mathbf{k}$ is the wave vector. For the non-equilibrium situation these functions are generalized to

$$
C_{c, k}\left(t_{w}+\tau, t_{w}\right)=\frac{1}{N} \sum_{j, l} \exp \left(i \mathbf{k} \cdot\left(\mathbf{r}_{j}\left(t_{w}+\tau\right)-\mathbf{r}_{l}(\tau)\right)\right.
$$

and

$$
C_{k}\left(t_{w}+\tau, t_{w}\right)=\frac{1}{N} \sum_{j} \exp \left(i \mathbf{k} \cdot\left(\mathbf{r}_{j}\left(t_{w}+\tau\right)-\mathbf{r}_{j}(\tau)\right)\right.
$$

(Note that these last equations are trivially generalized to multi-component systems.) For the present system the dependence of $F(k, t)$ and $F_{s}(k, t)$ on time and temperature have been discussed extensively in Refs. [8, 13]. In Fig. [t we show the time dependence of the corresponding quantities $C_{k}\left(t_{w}+\tau, t_{w}\right)$ and $C_{c, k}\left(t_{w}+\tau, t_{w}\right)$ for different waiting times $t_{w}$. The values of $t_{w}$ are 0 , i.e. immediately after the quench, and $t_{w}=10,100,1000,10000$, and 63100 time units and the final temperature $T_{f}=0.4$ (solid lines). In Fig. (1a we show $C_{k}\left(t_{w}+\tau, t_{w}\right)$ for $k=7.23$, the location of the first peak in the partial structure factor of the A-A correlation [8]. We see that the correlation function decays quite quickly as a function of $\tau$, which in view of the fact that we are at a very low temperature might be surprising. However, one should realize that the decay of the correlation function to zero does not imply that the system has relaxed to equilibrium but only that the particle configuration at the end of the measurement is uncorrelated with the configuration at the start. From the figure we see that with increasing value of the waiting time the decay of the curves occurs at longer and longer times. This observation can be rationalized by realizing that the driving force which leads to the decorrelation of the state at time $\tau$ from the state at time zero decreases with increasing waiting time $t_{w}$ since in the time between zero and $t_{w}$ the system had already the possibility to relax. This $t_{w}$ dependence of the curves is, however, only observed at long times. For short times the curves fall onto a master curve and leave this master curve only at a time which increases with $t_{w}$ (and is roughly proportional to $t_{w}$ [1]]). The time regime in which this master curve is observed is usually called "short time regime" whereas the time regime in which the curves show a significant $t_{w}$ dependence is called the "aging regime". In the short time regime the particles rattle in the cages formed by their nearest neighbors and this type of motion is thus not sensitive on the value of $t_{w}$ (if $t_{w}$ is not too small). Only for longer times the particles are able to leave this cage and the typical time scale for this process depends strongly on $t_{w}$.

We also mention that the oscillation seen in the curves at $\tau=1.0$ originates from the coupling of the system to the heat bath. Since at this time the velocities of all particles are swapped with the ones drawn from a MaxwellBoltzmann distribution at temperature $T_{f}$, the motion of the particles is, on average, slowed down or even reversed. Hence for a brief period the relaxation is slower than expected and thus the correlation curve decays slower. This effect is, however, only seen at short times and thus can be disregarded for large values of $\tau$.

It is also interesting to compare the relaxation behavior of the system in this non-equilibrium situation with the one in equilibrium. In Fig. Ga we have therefore also included the incoherent intermediate scattering function $F_{s}(k, t)$ in equilibrium (dashed lines) which was obtained in a simulation of Gleim et al. [13]. The dashed line is a curve that corresponds to the equilibrium relaxation dynamics of the system at $T=0.446$ (this was the lowest temperature at which the system could be equilibrated). For very short times, $\tau \leq 1.0$, this curve essentially coincides with the above discussed master curve in the short time regime. For times $\tau$ larger than 1.0 deviations are observed, which are not due to the mentioned oscillations. A careful inspection of the curves shows that the approach of the master curve and the dashed curve to the (quasi) plateau at intermediate times is quite different from each other in that the former shows a very slow approach whereas the one of the latter is quite fast.

Also at long times we find differences in the relaxation behavior. In equilibrium it has been demonstrated that the relaxation curves can be fitted very well by the so-called Kohlrausch-Williams-Watts (KWW) function, $A \exp \left(-\left(\tau / \tau_{\text {rel }}\right)^{\beta}\right)$, where $\tau_{\text {rel }}$ is the relaxation time and an exponent $\beta \leq 1[8$. From the figure we see that at long times the shape of the equilibrium and non-equilibrium curves are quite different and a more detailed analysis shows that a KWW function does not give a good fit to the data. What seems to be the same, however, (or at least very similar) is the height of the plateau, i.e. the so-called Edwards-Anderson parameter [21] or non-ergodicity parameter.

In Fig. $\$ \mathrm{~b}$ we show the relaxation curves for the collective function, i.e. $C_{c, k}\left(t_{w}+\tau, \tau\right)$ for the same waiting times. The wave-vector is now $k=9.60$, the location of the minimum in the partial structure factor of the A-A correlation [8]. Due to the collective nature of this correlation function the statistics is worse than the one for the self part but from the figure we see that also this observable can be studied with satisfactory accuracy. Since at this wave-vector the (equilibrium) value of the non-ergodicity parameter has a local minimum [8] it is expected that also the one for the non-equilibrium case is relatively small and from the figure we see that this is indeed the case. 


\section{B. Quantitative analysis of the relaxation at high temperatures}

In the following we will now analyze the relaxation behavior of the system in more detail. For this we start first with the dynamics at short times. Mean field calculations predict that for times at which the correlators are in the vicinity of the plateau, a time regime which in the equilibrium dynamics of glasses is called " $\beta$-relaxation regime, two power-laws are observed [7,22]. Denoting by $q_{\mathrm{EA}}$ the value of the Edwards-Anderson parameter, it is predicted that any time correlation function $C\left(t_{w}+\tau, t_{w}\right)$ should behave like

$$
C\left(t_{w}+\tau, t_{w}\right) \approx q_{\mathrm{EA}}+c_{a} \tau^{-a} \quad \text { if } \quad C \geq q_{\mathrm{EA}}
$$

and

$$
C\left(t_{w}+\tau, t_{w}\right) \approx q_{\mathrm{EA}}-c_{b}\left(\tau / \tau_{\alpha}\right)^{b} \quad \text { if } \quad C \leq q_{\mathrm{EA}}
$$

Here $\tau_{\alpha}$ is the typical relaxation time at long times and the exponents $a$ and $b$ are related by

$$
m \frac{\Gamma^{2}(1+b)}{\Gamma(1+2 b)}=\frac{\Gamma^{2}(1-a)}{\Gamma(1-2 a)} .
$$

The quantity $m$ in the last equation is the so-called fluctuation-dissipation-violation factor and will be discussed later in more detail. For the moment it is sufficient to know that it is expected to be a system universal number equal or less than 1.0. We also mention that the two power-laws of Eqs. (5) and (6) have been discussed for a long time and in great detail in the MCT of supercooled liquids and glasses [3, 1 . In order to see whether it is possible to see these power-laws in our data we have estimated the value of $q_{\mathrm{EA}}$ for $C_{k}\left(t_{w}+\tau, \tau\right)$ for $k=12.53$ and show in Fig. $5\left|C_{k}\left(t_{w}+\tau, \tau\right)-q_{\mathrm{EA}}(k)\right|$ in a double logarithmic plot for different waiting times. This value of $k$ is relatively large so that the value of $q_{\mathrm{EA}}$ is relatively small, 0.47. A small value of $q_{\mathrm{EA}}$ is useful if one wants to check for the presence of the power-law given in Eq. (5) whereas for the check of Eq. (6) a large value of $q_{\mathrm{EA}}$ is better. (These conclusions are reached from the analysis of equilibrium dynamics [23] but it can be expected that they hold also for the nonequilibrium case.) From the figure we recognize that it is indeed possible to see a power-law in the short time regime with an exponent around $0.42 \pm 0.05$ (bold solid line). In the discussion of Fig. 1 we said that in the late part of the $\beta$-relaxation regime the equilibrium curves and the non-equilibrium curves show a very similar time dependence and that the one of equilibrium is given by a power-law. The exponent of this power-law is around 0.63 [14.20]. If we assume the relation (7) to hold true one therefore obtains $m \approx 0.57$. Due to the relatively large error of the exponents $a$ and $b$ this number also has a significant error.

We have also checked for the presence of the power-law for other values of $k$, as well as for the collective correlation function $C_{c, k}\left(t_{w}+\tau, t_{w}\right)$ and found that all of them show such a time dependence with an exponent which is compatible with 0.42 . Unfortunately, however, the exact determination of the exponent is rather difficult, since a change of the (unknown) value of $q_{\text {EA }}$ will lead to a change in the exponents also. In order to avoid this problem to some extend we use a trick which has proved to be useful in the context of the analysis of equilibrium data (see, e.g., 24,25]). For the equilibrium case MCT predicts that in the $\beta$-relaxation regime the so-called factorization property holds 3 , 1 . This means that any time correlation function $\phi(t)$ can be written as $\phi(t)=q_{\mathrm{EA}}(\phi)+h_{\phi} G(t)$, where $h_{\phi}$ is a constant and the whole time dependence is given by the $\phi$-independent, i.e. system universal, function $G(t)$. If we assume that a similar relation holds also in the non-equilibrium situation we have

$$
\phi\left(t_{w}+\tau, t_{w}\right)=q_{\mathrm{EA}}(\phi)+h_{\phi} G\left(t_{w}+\tau, \tau\right) .
$$

We see that if the exponents $a$ and $b$ as well as the quantity $m$ are independent of the observable that then Eqs. (5) and (6) are indeed compatible with such an Ansatz. From Eq. (\&) it follows immediately that if $\tau^{\prime}$ and $\tau^{\prime \prime}$ are two arbitrary times in the $\beta$-relaxation regime, the ratio

$$
R_{\phi}\left(t_{w}+\tau, t_{w}\right)=\frac{\phi\left(t_{w}+\tau, t_{w}\right)-\phi\left(t_{w}+\tau^{\prime \prime}, t_{w}\right)}{\phi\left(t_{w}+\tau^{\prime}, t_{w}\right)-\phi\left(t_{w}+\tau^{\prime \prime}, t_{w}\right)}
$$

is independent of $\phi$ if $\tau$ is in the $\beta$-relaxation regime also. In Fig. 6 we plot this ratio for different choices of $\phi$. These are $C_{c, k}\left(t_{w}+\tau, t_{w}\right)$ for $k=6.52,7.23,9.6$, and 12.53 as well as $C_{k}\left(t_{w}+\tau, t_{w}\right)$ for the same wave-vectors and also $k=2.0$ and $k=3.0$. The value of $t_{w}$ is kept fixed at $t_{w}=63100$. From the figure we see that in the $\beta$-relaxation regime the different curves collapse nicely onto a master curve, thus demonstrating the validity of the factorization property. That this result is by no means trivial can be recognized from the fact that for very short and very long times the different curves do not fall onto a master curve at all. From the existence of the master curve in 
the $\beta$-relaxation regime we thus come to the conclusion that, within the accuracy of our data, in this time regime the time dependence of the relaxation is governed by one single system universal function $G\left(t_{w}+\tau, t_{w}\right)$. From the results shown in Fig. 5 we see that this function is compatible with power-law of the form given by Eq. (5).

As we will see later, it is expected that the quantity $m$ depends on the value of $T_{f}$ [ [7]. Therefore one might conclude that also the exponent $a$ and $b$ depend on temperature. From making fits to the master curves in the $\beta$-relaxation regime of our data, see Fig. 5, it is hard to conclude whether or not such a dependence is indeed present since the choice of the non-ergodicity parameter affects the values of the exponents also. Therefore we have calculated the ratios $R_{\phi}\left(t_{w}+\tau, t_{w}\right)$ also for lower values of $T_{f}, T_{f}=0.3$ and 0.1 , and found that the curves for the different observables do indeed fall onto a master curve also. More important is the observation that within the accuracy of our data this master curve does not depend on $T_{f}$, thus giving evidence that the function $G\left(t_{w}+\tau, t_{w}\right)$ is only a weak function of $T_{f}$.

We also mention that mean-field calculations lead to the expectation that the quantities $a, b$ and $m$ in Eq. (7) do depend on the final temperature. (This is in contrast to the equilibrium MCT where $m=1$ and $a$ and $b$ are independent of temperature.) In Sec. $\mathrm{VG}$ we will show that $m$ shows a significant dependence on the final temperature in that it becomes smaller with decreasing $T_{f}$. Thus that result might seem to be in contradiction with the fact that $G\left(t_{w}+\tau, t_{w}\right)$ seems to depend only weakly on $T_{f}$. The resolution of this apparent problem is that if we assume $b$ to be constant that a decrease of $m$ can be compensated by an increase of $a$. It is, however, simple to see that if $m$ is small $a$ has to be close to 0.5 and that a variation of $m$ can be compensated by a very small change in $a$, i.e. without leading to an appreciable change in $G\left(t_{w}+\tau, t_{w}\right)$.

Having analyzed the relaxation behavior of the system in the $\beta$-relaxation regime we now investigate the one of the $\alpha$-regime, i.e. the relaxation of the system at long times. When we discussed the time correlation functions in Fig. Ala we already mentioned that in this regime the relaxation differs from the one in the equilibrium situation in that the time correlation functions can not be described well by the KWW-law. In Fig. Fa we show the same correlation

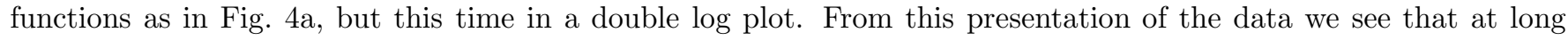
times the out-of-equilibrium curves show a power-law. The exponent is around 0.4 and is, within the accuracy of our data, independent of $t_{w}$. A similar time dependence has also been found in simulations of spin-glasses [26], although in that case the exponent was significantly smaller, and is thus not unusual for the aging dynamics.

The curves in Fig. $7 \mathrm{a}$ are for the wave-vector $q=7.23$, the location of the main peak in the static structure factor. In Fig. 7 $7 \mathrm{~b}$ we show the same type of correlation function for different wave-vectors and $t_{w}=1000$. From the main figure we see that the dependence of $C_{k}$ on $k$ is quite pronounced, in that e.g. the height of the plateau increases quickly with decreasing wave-vector. Such a dependence can be understood at least qualitatively by recalling that in equilibrium the wave-vector dependence of the Edwards-Anderson parameter is very similar to a Gaussian, and that at short times and long waiting times the time correlation functions do not depend on $t_{w}$ (see Fig. 4), i.e. show a quasi-equilibrium behavior. From Fig. $7 \mathrm{~b}$ we see that a rough estimate of $q_{k}$ is given by the value of $C_{k}\left(t_{w}+\tau, t_{w}\right)$ at $\tau=20$ and we find that this quantity does indeed show a Gaussian like dependence on $k$.

In the inset of the figure we show the same correlation functions in a double logarithmic plot. From this graph we recognize that for all wave-vectors the long time dependence of the functions are compatible with a power-law and that the exponent depends on the wave-vector in that it decreases with decreasing $k$.

Since at long times $C_{k}$ shows a power-law dependence it is not possible to define a relaxation time. However, from the figure it becomes clear that the relaxation of the system is much faster on small length scales than on large ones. It is instructive to recall that for a diffusion process the relaxation time depend on the wave-vector like $k^{-2}$. A comparison of the curves for $k=3.0$ and $k=6.5$ shows that the time needed to decay to $50 \%$ of the initial value differs by almost a factor of 100, thus much more than the factor of 4 expected for a diffusive process, or the factor found in the relaxation dynamics of supercooled liquids in equilibrium. This shows that during the aging process the relaxation at long times is indeed very different from the one in supercooled liquids.

We now turn our attention to the time and waiting time dependence of the two-time correlation functions at long times. Within mean-field theory it is expected that for systems which show a discontinuous dependence of the nonergodicity parameter on temperature, which is the case for the structural glass studied here, this dependence can be written (in the limit of long waiting times) as

$$
C\left(t_{w}+\tau, t_{w}\right)=C_{\mathrm{ST}}(\tau)+C_{\mathrm{AG}}\left(\frac{h\left(t_{w}+\tau\right)}{h\left(t_{w}\right)}\right) .
$$

Here $C_{\mathrm{ST}}(\tau)$ is the time dependence of $C\left(t_{w}+\tau, t_{w}\right)$ at short times which is supposed to be independent of $t_{w}$ (in agreement with our findings, see Fig. (1) and to decay quickly to zero. $C_{\mathrm{AG}}$ is a function whose form depends on $C\left(t_{w}+\tau, t_{w}\right)$ and $h(t)$ is a monotonously increasing function of the argument. The interesting point of this equation is that the whole $\tau$ and $t_{w}$ dependence of the aging regime enters only through the combination $h\left(t_{w}+\tau\right) / h\left(t_{w}\right)$. Apart from Eq. (10), not much more is known about the $\tau$ and $t_{w}$ dependence of $C\left(t_{w}+\tau, t_{w}\right)$. 
In order to gain more insight into this dependence we investigate whether the function $h(t)$ is independent of the correlation function $C\left(t_{w}+\tau, t_{w}\right)$. The presence of such a dependence can, e.g., be checked by plotting a time correlation function $C_{k^{\prime}}\left(t_{w}+\tau, t_{w}\right)$ versus a different time correlation function, e.g., $C_{k}\left(t_{w}+\tau, t_{w}\right)$, i.e. by making a parametric plot with time $\tau$ as the running parameter. If $h(t)$ is independent of the correlation function considered, it is easy to see that in such a parametric plot the curves corresponding to different waiting times will fall on top of each other. (This holds true only if the time dependence of $C_{S T}(\tau)$ can be neglected, i.e. at long times.)

In Fig. 8 we show such parametric plots for the waiting times $t_{w}=0,10,100,1000,10000$, and 63100 time units. The independent variable (abscissa) is $C_{k}\left(t_{w}+\tau, t_{w}\right)$ for $k=7.23$ and the dependent variables (the ordinate) are the same correlation function for different values of $k$. Let us focus first on $k=2.0$. From the graph we recognize that at short times (corresponding to large values of $C_{k}$ and $C_{k^{\prime}}$ ) the parametric plot is independent of $t_{w}$, as it would be expected for the equilibrium case. However, for times at which the correlation functions have fallen below their Edwards-Anderson parameters a waiting time dependence is seen. Qualitatively the same behavior is found for the other values of $k^{\prime}$. From this observation we hence conclude that if the Ansatz in Eq. (10) is correct, then the function $h(t)$ does depend on the observable, i.e. in this case on the wave-vector. We also mention that qualitatively the same results are obtained for the different values of $T_{f}$.

The time dependence of the function $h(t)$ can be used to distinguish between different theoretical models to describe the aging dynamics. E.g. the droplet model of Fisher and Huse [27] predicts $h(t)$ to be of the form $h(t)=\log (t)$. In Ref. 28] it was argued that the present Lennard-Jones model showed this type of aging dynamics, a conclusion which was not confirmed by the present authors [29]. The reason for this discrepancy remains still unresolved. In order to settle this issue we have attempted to determine the function $h(t)$ from our data without making reference to any model, i.e. functional form of $h(t)$. For this we assumed that at long times the correlation functions are given by the second term in Eq. 10. Starting from the initial guess $h(t)=\log (t)$ we plotted the correlation function versus $h\left(t_{w}+\tau\right) / h\left(t_{w}\right)$ for all values of $t_{w}$, which gave at long times a clustering of the curves. By iteratively bending $h(t)$ in a continuous way by small amounts and subsequently making the scaling plot we attempted to generate a collapse of the curves. The outcome of this procedure is shown in Fig. 9 for the case $C_{k}$ with $k=7.23$. (For the sake of clarity only the curves for $t_{w}=0,10,100,1000,10000$, and 63100 time units are shown, although more waiting times were considered to determine the master curve. Also, in order to expand the abscissa we have subtracted 1.0 from $h\left(t_{w}+\tau\right) / h\left(t_{w}\right)$ and plot its logarithm.). From this figure we see that the it is indeed possible to obtain a satisfactory scaling of the curves at long times. The function $h(t)$ which was obtained by the procedure described above is shown in the inset. We see that to a first approximation it is close to a logarithm, but that significant deviations are present.

Also included in the figure are the curves for different values of the wave-vector. We see that in these cases the curves for the different waiting times do not collapse nicely at long times. Thus this is further evidence that the function $h(t)$ depends on the observable considered. We also mention that although we have determined the optimal shape of $h$ also for the other wave-vectors, it is difficult to compare these different functions with each other. The problem is, that if $h(t)$ is an optimal function that for example also $h(t)^{\alpha}$ (with arbitrary $\alpha$ ) is an optimal function, i.e. there is no unique representation of $h(t)$.

\section{Relaxation at low temperatures}

Essentially all the results discussed so far were obtained for a final temperature $T_{f}=0.4$. This is only slightly $(10 \%)$ less that the mode coupling critical temperature for the system, $T_{c}=0.435$ [8]. A markedly different behavior in the relaxation is observed for temperatures much lower than $T_{c}$, as illustrated in figure 10, which shows the results for $C_{k}\left(t_{w}+\tau, t_{w}\right)$ after a quench to $T_{f}=0.1$. The values of the waiting times and of the wave-vector $k$ are the same as in figure $\mathrm{\theta}\left(T_{f}=0.4\right)$. At short times $\tau$, the dependences are qualitatively similar for the two final temperatures. The only difference is the increase in the plateau value when the temperature is lowered. This increase is easily understood from the fact that the amplitude of the vibrational motion about an equilibrium position decreases when $T$ decreases. From harmonic theory a linear dependence of $1-q_{E A}$ on $T$ at low temperatures can be expected, and such a dependence is indeed compatible with our results.

For large values of $t_{w}$ and $\tau$, the behaviour for $T_{f}=0.1$ is strikingly different from what was observed at $T_{f}=0.4$. Instead of decaying rapidly to zero for $\tau>t_{w}$, the correlators level off and appear to display an additional plateau at a value of $C$ smaller than $q_{E A}$.

In order to get some insight into this surprising behavior, we have studied separately the correlation functions obtained for various samples prior to averaging. Looking at the data shown in fig. 10 for some of the samples, it appears that the decay to this second plateau is triggered by a large amplitude and rather sudden drop of the correlation function, that, depending on the sample, takes place typically $10^{3}$ to $10^{4}$ time units after the quench (see inset of Fig. 10). An analysis of the configurations shows that this sudden drop is related to a very collective dynamical 
event, in which a substantial fraction of the particles (10\%) move by a rather small amount, typically 0.1-0.5 $\sigma_{A A}$. Such motions can be understood from the fact that a deep quench leaves the system in a highly stressed configuration, so that the relaxation first proceeds through large "earthquake like" events, that release the local stress significantly. Only at longer times the aging dynamics crosses over to the very smooth process observed at higher values of $T_{f}$.

We emphasize that these events do not seem to be related to the hopping like motion of the particles which is observed in the deeply supercooled state above the glass transition temperature [30]. In that case only very few (2-3) particles are involved and during the jump these particles move on the order of one nearest neighbor distance. In contrast to this, the "catastrophic events" during the aging are very collective (50-100 particles) and the particles move only about 10-30\% of the typical nearest neighbor distance (as can be inferred from the self part of the van Hove correlation function). A typical event is shown in Fig. 11 where we show the particles just before the event (spheres) and their location after the event (tip of arrows). From this figure we recognize that in such an event the stress is released along a surface or even a line through the sample and not through the motion of the particles in a (bulk-like) three dimensional blob.

Obviously, the catastrophic events that cause the decay of the correlations in this situation are rather difficult to average over, and a very large number of samples would be required to obtain a reasonable statistical accuracy. Moreover, it is quite likely that these events correspond to a transient, so that the second plateau we observe is not really representative of the asymptotic behavior. Interestingly, however, rather similar shapes of the time correlation functions were observed by Bonn and coworkers [31] in their dynamical light scattering studies of aging in clay (laponite) suspensions. In that case the height of the second plateau was found to steadily increase with waiting time, confirming the transient nature of the effect.

We also mention that the occurence of these events are related to the fact that the typical configuration at high temperature $\left(T_{i}=5.0\right)$ are quite different from the ones towards the system evolves to at $T_{f}$, thus giving rise to large stresses. Since in strong glass formers (e.g. $\mathrm{SiO}_{2}$ ) the structure has a much weaker temperature dependance than in fragil eglassformers such as the present system, it can be expected that in the aging dynamics of the former no such "catastrophic events" should be observed.

\section{NONEQUILIBRIUM RESPONSE AND FLUCTUATION DISSIPATION RATIO}

\section{A. Definition and measurement of the nonequilibrium response}

One of the crucial points that was derived from the solution of the dynamical equations in mean field models of spin glasses is the fact that, in a nonequilibrium system, the Fluctuation Dissipation Theorem (FDT) is violated in a nontrivial way. We will first briefly recall the main results from mean-field theories of spin glasses, and then discuss how the violation of FDT can be quantified in our system.

Let us consider an observable $A$ whose normalized autocorrelation function is denoted by $C$. If $H$ denotes a field conjugate to $A$, the response of $A$ to $H$ is defined as $R\left(t, t^{\prime}\right)=\frac{\delta A(t)}{\delta H\left(t^{\prime}\right)}$. In an equilibrium system, this response function is invariant under time translation, i.e. $R\left(t, t^{\prime}\right)=R\left(t-t^{\prime}\right)$, and is related to the correlation function by the fluctuation dissipation theorem, $R\left(t, t^{\prime}\right)=\frac{1}{k_{B} T} \frac{\partial C\left(t, t^{\prime}\right)}{\partial t^{\prime}}$. Out of equilibrium, this relation does not hold any more, and a "Fluctuation Dissipation Ratio" (FDR) $X\left(t, t^{\prime}\right)$ can be defined as

$$
R\left(t, t^{\prime}\right)=\frac{1}{k_{B} T} X\left(t, t^{\prime}\right) \frac{\partial C\left(t, t^{\prime}\right)}{\partial t^{\prime}} .
$$

Much attention has been devoted to the asymptotic behavior of this fluctuation dissipation ratio. In particular, it has been shown [32] that in the asymptotic limit $t, t^{\prime} \rightarrow \infty$, the fluctuation dissipation ratio in mean field models of spin glasses, as well as in coarsening systems, becomes a non-singular function of $C\left(t, t^{\prime}\right)$, i.e. $X\left(t, t^{\prime}\right)=x\left(C\left(t, t^{\prime}\right)\right)$. A direct consequence from this is that the more easily accessible integrated response

$$
M\left(t_{w}+\tau, t_{w}\right)=\int_{t_{w}}^{t_{w}+\tau} R\left(t_{w}+\tau, t\right) d t
$$

can be written as a continuous function of $C$

$$
M\left(t, t^{\prime}\right)=M(C) \int_{C}^{1} x(c) d c
$$


From a practical point of view, the nontrivial consequence of these statements is that a parametric plot of $M\left(t_{w}+\right.$ $\left.\tau, t_{w}\right)$ versus $C\left(t_{w}+\tau, t_{w}\right)$ (with $\tau$ as the parameter) should, for long times, converge towards a master curve, independent of the waiting time. Such a behavior has been observed in a number of spin glass simulations [33 35.

In order to test whether the same property holds in structural glasses, we have to devise a way of measuring the response function associated to $C_{k}\left(t, t^{\prime}\right)$, which is the only correlation function that can be obtained with a reasonable accuracy in the aging regime. The procedure we use is the following. A fictive "charge" $\epsilon= \pm 1$ is assigned randomly to each particle. An additional term of the form $\sum_{j} \epsilon_{j} V\left(\mathbf{r}_{\mathbf{j}}\right)$, where $V(\mathbf{r})=V_{0} \cos (\mathbf{k} \cdot \mathbf{r})$ is a small $\left(V_{0}<k_{B} T\right)$ external potential, is then added to the Hamiltonian. It is then easy to check that, if one averages over several realizations of the random charge distribution, the time-correlation function of the observable $A_{k}=\sum_{j} \epsilon_{j} \exp \left(i \mathbf{k} \cdot r_{j}(t)\right)$ is the incoherent scattering function $C_{k}$. The procedure to generate the response function associated to $C_{k}$ is thus straightforward: For a given realization of the random charge distribution, the system is equilibrated at a high temperature $\left(T_{i}=5.0\right)$, and quenched at $t=0$ to the desired final temperature $T_{f}$. The evolution is followed with the field $V(\mathbf{r})$ off until a waiting time $t_{w}$, then the field is switched on and the response $A_{k}\left(t_{w}+\tau, t_{w}\right)$ is monitored. The same procedure is repeated for several ( 7 to 10 ) realizations of the charge distribution, in order to get the response function. The quantity we obtain by this procedure is then an integrated response function $M\left(t_{w}+\tau, t_{w}\right)$ (Eq. 12), since

$$
\begin{aligned}
\left\langle A_{k}\left(t_{w}+\tau, t_{w}\right)\right\rangle & =V_{0} \int_{t_{w}}^{t_{w}+\tau} R\left(t_{w}+\tau, t\right) d t \\
& =V_{0} M\left(t_{w}+\tau, t_{w}\right) .
\end{aligned}
$$

We have checked that this procedure indeed yields the correct response function by checking the fluctuation dissipation theorem in an equilibrium system. In that case, a slightly different procedure was used, in the sense that we monitored the decay of the response after switching off the field. The fluctuation dissipation theorem implies that this decay is directly proportional to the correlation function, a result that is illustrated in fig. 12. From this figure we see that the quality of the response data is significantly poorer than the one for the correlation data. This is due to the fact that the latter can be averaged over time origins and wave-vector directions, hence improving drastically the statistical accuracy.

\section{B. Results for the response and the fluctuation dissipation ratio}

For the nonequilibrium case typical results for the integrated response function are shown in Fig. 13 , for $T_{f}=0.4$, $k=7.25$ and two values of the waiting time. As expected, the response, like the correlation, gets slower as the waiting time increases. As in the equilibrium case, the quality of the response data is poorer than that of the correlation data. In that case, the average over wave-vector directions in obtaining the correlation data probably explains this difference. Despite this noise it can be seen from the figure that at long times the curve for the response lies above the one for the correlation function, i.e. that the response is smaller than expected from the FDT.

As explained above, mean field theories of spin glasses suggest that interesting information can be obtained from a parametric plot of $M\left(t_{w}+\tau, t_{w}\right)$ versus $C\left(t_{w}+\tau, t_{w}\right)$ at fixed $t_{w}$. Such a plot is shown in figures 14 (a-c) for three values of the final temperature, $T_{f}=0.4, T_{f}=0.3$ and $T_{f}=0.1$. The wave-vector is $k=7.25$. For each temperature, results obtained at different waiting times are shown. In spite of the scatter in the data, the figures are clearly compatible with the existence of a master curve independent of the waiting time. Obviously, the quality of the data does not allow a quantitative analysis of this master curve. We can nevertheless argue that two different regimes can be distinguished. For high values of the correlation ( $C_{k}$ close to unity), the parametric plot is essentially a straight line with slope -1 . A slope of -1 would be observed in an equilibrium system obeying FDT. At lower values of the correlation, a clear deviation from this FDT slope is observed. Although other fits are certainly possible, we can describe the parametric plot as consisting of two straight lines, one with slope -1 and one with a negative slope $-1<-m<0$. The reason for choosing such a description and the corresponding interpretation will be discussed in the next section.

A similar analysis was performed for another value of the wave-vector $(k=3.0)$. Very similar results are obtained, and the slope of $m$ of the non-FDT part in the parametric plot seems to be independent of the wave-vector.

Before discussing the results, we mention that the results for this parametric plot are very sensitive to the linearity of the response. Hence we always checked carefully that our results were independent of the amplitude of the applied field. In practice, applying a stronger field does not affect the FDT part of the parametric plot, but tends to affect strongly the second part, in that it leads to a decrease in the parameter $m$ that describes the non-FDT part of the curve. 


\section{Discussion}

The importance of the fluctuation dissipation ratio $x(C)$ in glassy systems was first recognized in the context of the mean field theory of spin glasses [32], when it appeared that this FDR was intimately related to the nature of ergodicity breaking in the system. The nature of this relationship was recently clarified by Franz et al. 36], who showed that the asymptotic value of the FDR could be quite generally expressed in terms of the probability distribution $P(q)$ of overlaps between replicas introduced by Parisi [37] through

$$
x(C)=\int_{0}^{C} P(q) d q .
$$

This relation between a purely static quantity, $P(q)$, and a dynamical one, $x(C)$, is extremely powerful, since it implies that a nontrivial information on the nature of phase space may be encoded in the nonequilibrium time dependent behavior.

If we accept equation (16), a small number of possible scenarios are documented in the literature [38]. For systems with continuous step replica symmetry breaking, $P(q)$ is a continuous function, and so is $x(C)$. For systems with one step replica symmetry breaking like $p$-spins systems $(p>3), P(q)$ consists of two $\delta$-functions at $q=0$ and $q=q_{E A}$, so that $x(C)=1$ for $C>q_{E A}$ and $x(C)=m<1$ for $C<q_{E A}$. Finally simple coarsening systems, like the Ising system below its ferromagnetic transition, have an essentially trivial $P(q), P(q)=\delta\left(q-M^{2}\right)$, where $M=M(T)$ is the magnetization. Therefore, in that case the FDR is 1 if $1>C>M^{2}$, and 0 if $M^{2}>C$ 39 41].

Within this theoretical framework, we have to find which scenario is compatible with the results presented in the last section. From Figs. 14, it would appear that the most likely scenario is that of a system with one step replica symmetry breaking, for which $x(C)$ is a stepwise constant function. In that case the parametric plot consists in two straight lines, one with slope -1 and one with slope $-1<-m<0$ (bold straight lines).

Obviously the asymptotic nature of the results can be questioned. However, the fact that we obtained an essentially $t_{w}$ independent plot is already an indication that we are approaching the asymptotic limit. It could also be that preasymptotic effects cancel out when the parametric plot is used. Indeed, such a plot is even obtained for $T_{f}=0.1$, where we have seen that strong "catastrophic" events influence the relaxation. Finally, we mention that in systems in which preasymptotic effects have been observed and studied (mostly domain growth models) [38,41], they very clearly show up in the parametric plot as a $t_{w}$ dependence of the crossover region between the FDT part and the non-FDT region. The overall shape of the parametric plot is not affected much. Such a dependence could then explain the fact that in our data, the crossover between the FDT part and the non-FDT part takes place at a value of $C$ smaller than the plateau value $q_{E A}$ in the correlation function, a feature which contradicts theoretical expectations. The tendency of the curves to "overshoot" in the crossover region, perceptible in Fig. 14, is reminiscent of the observations made in reference [38], and could also be a transient effect. In that paper it was argued that, e.g., in a coarsening system with some defects the de-pinning of a domain wall from a defect will give rise to a strong enhancement of the response just after the event. Since the "catastrophic" events correspond to a violent and, most likely, pre-asymptotic release of the stress, it is reasonable to assume that also in this case the response will be larger than expected.

If we accept that our parametric plots correspond to the one step replica symmetry breaking case, we can extract from the data an estimate for the slope $-m$ of the non-FDT part. The resulting slopes are given by: $T_{f}=0.4$, $m=0.62 \pm 0.05 ; T_{f}=0.3, m=0.45 \pm 0.05 ; T_{f}=0.1, m=0.2 \pm 0.1$. As mentioned above, these results appear to be independent of the wave-vector. Within the accuracy of our data, they are compatible with a linear dependence of $m$ on $T_{f}$, quite similar to that found by Parisi [12] for a soft-sphere system. We also note that the value found for $T_{f}=0.4$ is compatible with the result $m=0.57$ that was extracted from a scaling analysis of the correlation functions alone.

It is interesting to analyze our results for $m$ using the "effective temperature" concept introduced in reference [42]. (For a different approach to the effective temperature see the papers of Nieuwenhuizen [43]). In this approach, an effective "fluctuation dissipation temperature" $T_{\text {eff }}$ is defined as $T_{\text {eff }}=T / m$, where $T$ is the actual external temperature. Crudely speaking, the "fast" degrees of freedom (those that correspond to the rapid decay of $C$ to its plateau value) are at equilibrium with the thermostat at temperature $T$, while the slow degrees of freedom, which govern the aging behavior, are at equilibrium at a higher temperature. Then the concept of effective temperature can be thought as a rationalization of the older "fictive temperature" concept [44]. Within this interpretation, a linear dependence of $m\left(T_{f}\right)$ on $T_{f}$ corresponds to a constant effective temperature. It is therefore quite natural to expect first that $m\left(T_{f}\right)$ should be equal to $T_{f} / T_{c}$, where $T_{c}$ is the mode coupling critical temperature, since systems cooled below $T_{c}$ fall out of equilibrium at $T_{c}$. This result was proposed by Parisi [12] on the basis of his simulations in a soft sphere system. Our results do not corroborate such a simple interpretation. The "effective temperature" in our aging system is substantially larger than $T_{c}$, typically $T_{\text {eff }} \simeq 0.7$. Our results here are similar those of Alvarez et al. [45] for the $p$-spin model in that these authors found for a temperature a bit below $T_{c}$ a value of $m$ which is 
significantly smaller than 1 . Indeed, the "ideal" result $m\left(T_{f}\right)=T_{f} / T_{c}$ can only be expected to hold for systems that would be cooled infinitely slowly, so that they would remain in equilibrium down to $T_{c}$, and for which ergodicity restoring hopping processes can be completely neglected. For a system that is cooled with a finite cooling rate, it is not surprising to find an effective temperature which is above $T_{c}$.

\section{CONCLUSIONS}

In this paper, we have presented a detailed numerical study of the out of equilibrium relaxation ("aging") of a simple glass forming system. The time scales we have investigated are those allowed by the current possibilities of Molecular Dynamics simulations, typically $10^{-8}-10^{-7} \mathrm{~s}$. We will now try to briefly summarize the main conclusions that can be inferred from these numerical results.

First, it appears that static quantities are, on these time scales, only very weakly dependent of time. By static (or "one time") quantities we mean, as usual, those quantities that can be obtained from the knowledge of a single configuration of the system. In particular, we have shown that the pair correlation functions (and hence all the derived quantities like the energy or the pressure) appear to equilibrate very quickly after the quench. These onetime quantities show some sensitivity to the quench history and to the final temperature and we find that to a first approximation they correspond to configurations which are very close to the critical surface of (equilibrium) modecoupling theory. Hence we have evidence that for this model and within the time span of the simulation the system is not able to penetrate this surface and hence remains in the liquid like part of configuration space.

In contrast to this weak dependence, the two-time correlation functions are very sensitive to the aging time. When the quench temperature is relatively high (i.e. still close to the mode-coupling critical temperature that was identified for our system), the waiting time dependence of these functions can be described in rather simple terms. Functions obtained for various $t_{w}$ can be rescaled on a master curve, and an analysis of this curve yields results that are compatible with the predictions of mean field ("mode-coupling") theories of the glass transition formulated for the " $p$-spin" spin glass models.

At lower final temperatures, the behaviour of the two-time correlation functions is much more complex. The relaxation is largely dominated by "catastrophic events" that involve a small, but collective, displacement of a large number of particles. These infrequent and large events are very difficult to average over, so that the statistical quality of the data for the correlation functions is rather poor. Due to the limited time range of the simulations, we do not know whether these events are transients effects releasing some initial stresses due to the quench, or constitute a genuine characteristic of low temperature relaxation.

The flexibility of MD simulations allows an independent measurement of the two-time response functions, which at equilibrium would be related to the correlation functions through the fluctuation dissipation theorem (FDT). A parametric representation of the response versus the correlation allows to clearly distinguish between a "quasiequilibrium" region in which the FDT holds, and a second regime in which it is violated. An essential feature is that this parametric plot does not (or, at most, very weakly) depend on waiting time, so that the "FDT" and "non-FDT" regimes do not correspond to time windows but rather to correlation windows. This remarkable feature, predicted by mean field theories of spin glasses to hold in the asymptotic limit, is observed here for structural glasses at finite times. This is likely related to the formal similarities between the mode-coupling theories of structural glasses, which are known to describe quite well the equilibrium behaviour of our system, and these mean field models of spin glasses. As the response/correlation relationship observed for our model resembles the one observed in spin systems with one step replica symmetry breaking, one may speculate that the phase space structures in both systems are also similar. If this is the case, a quite appealing scenario for the glass transition can be devised, that reconciles some of the old ideas of the Adams-Gibbs approach with the more modern mode-coupling scenario, as discussed in detail in reference 46.

Unfortunately, we presently do not have at our disposal a quantitative theory of nonequilibrium behaviour in structural glasses. The theoretical framework currently provided by mean-field theories of spin glasses can be considered as a schematic model, and hence does not allow comparisons beyond the qualitative level. At this level, we find that the general behaviour of our system is in quite good agreement with this schematic model. The agreement (and disagreements) are somewhat reminiscent of what is observed when comparing equilibrium data with predictions of schematic mode-coupling theories.

Finally, it is natural to inquire about the relevance of a work on aging phenomena based on a method that is limited to time scales smaller than $10^{-7} \mathrm{~s}$ to the aging effects observed on much longer time scales. The general picture we obtain provides, however, some support for a scenario 46] that may be of general relevance for much longer time scales. In this scenario, the critical temperature $T_{c}$ of mode coupling theory is associated to the dynamical freezing temperature of mean field spin models. In a purely mean field (or, in other words, ideal mode-coupling) situation, 
the system would be out of equilibrium at all temperatures below $T_{c}$. Of course, real systems can still be equilibrated below $T_{c}$, as "hopping processes" allow a relaxation that is not described by mode-coupling theory (at least in its ideal version). Hence in real experiments (or in our system, if we could allow for longer simulation times) these systems will show only interrupted aging, i.e. aging for short waiting times and no aging at long waiting times. However, we have shown that if the system was artificially driven into a nonequilibrium situation, its "short time aging" can be described reasonably well within the framework of mean-field/mode-coupling theories. We may here draw a parallel with the fact that, in equilibrium, below $T_{c}$, when the $\alpha$ (terminal) relaxation becomes dominated by hopping processes, the $\beta$ (intermediate times) relaxation is still very well accounted for by ideal mode coupling theory [3, 4. It is then quite tempting to speculate that for any "fragile" system (i.e. a system in which hopping processes are not too strong, so that mode coupling theory accounts well for the dynamics close to $T_{c}$ ) quenched below its glass transition, the mean-field/mode-coupling description will be relevant for a large fraction of the aging process. This should be particularly relevant in systems where hopping processes are known to be weak, like colloidal suspensions.

\section{ACKNOWLEDGMENTS}

We acknoweledge fruitful discussions with L. Cugliandolo, J. Kurchan and A. Latz. This work was supported by the Pôle Scientifique de Modélisation Numérique at ENS-Lyon, and by the Deutsche Forschungsgemeinschaft under SFB262.

[1] For a recent review see the series of papers in Science, 267, 1924 (1995) and the Proceedings of Third International Discussion Meeting on Relaxations in Complex Systems, Eds. K. L. Ngai, E. Riande, M. D. Ingram, J. Non-Crystal. Solids 235-237 (1998).

[2] See, e.g., J.-L. Barrat and M. L. Klein, Annu. Rev. Phys. Chem. 42, 23 (1991); W. Kob, p. 1 in Annual Reviews of Computational Physics Vol III, Ed. D. Stauffer (World Scientific, Singapore, 1995); W. Kob, J. Phys.: Condens. Matter 11 R85 (1999).

[3] W. Götze, p. 287 in Liquids, Freezing and the Glass Transition Eds.: J. P. Hansen, D. Levesque and J. Zinn-Justin, Les Houches. Session LI, 1989, (North-Holland, Amsterdam, 1991); W. Götze and L. Sjögren, Rep. Prog. Phys. 55, 241 (1992); W. Kob, p. 28 in Experimental and Theoretical Approaches to Supercooled Liquids: Advances and Novel Applications Eds.: J. Fourkas, D. Kivelson, U. Mohanty, and K. Nelson (ACS Books, Washington, 1997).

[4] For a recent review see, e.g., W. Götze, J. Phys. Condens. Matter 11, A1 (1999).

[5] L. C. E. Struik, Physical Aging in Amorphous Polymers and Other Materials (Elsevier, Amsterdam, 1978); G. B. McKenna, p. 311 in Comprehensive Polymer Science, Vol. 12, Polymer Properties, Eds.: C. Booth and C. Price (Pergamon, Oxford, 1989).

[6] E. Vincent, J. Hamman, M. Ocio, J.-P. Bouchaud, and L.F. Cugliandolo in Proceedings of the Sitges Conference on Glassy Systems, Ed.: E. Rubi (Springer, Berlin, 1996).

[7] J.-P. Bouchaud, L.F. Cugliandolo, J. Kurchan, and M. Mézard, Physica A 226, 243 (1996); J.-P. Bouchaud, L.F. Cugliandolo, J. Kurchan and M. Mézard in Spin Glasses and Random Fields, Ed.: A.P. Young (World Scientific, Singapore, 1998); preprint condmat/9511042.

[8] W. Kob and H. C. Andersen, Phys. Rev. E 53, 4134 (1995); ibid. 51, 4626 (1995); Phys. Rev. Lett., 73, 1376 (1994).

[9] R. L. Leheny and S. Nagel, Phys. Rev. B 57, 5154 (1998).

[10] N. E. Israeloff and T. S. Grigera, Europhys. Lett. 43, 308 (1998); T. S. Grigera and N. E. Israeloff, preprint condmat/9904351

[11] W. Kob and J.-L. Barrat, Phys. Rev. Lett. 78, 4581 (1997); Physica A 263, 234-241 (1999); J.-L. Barrat and W. Kob, Europhys. Lett. (1999) in press.

[12] G. Parisi, Phys. Rev. Lett. 79, 3660 (1997); J. Phys.: Math Gen. 30, L765 (1997). ibid. 30, 8523 (1997).

[13] T. Gleim, W. Kob, and K. Binder, Phys. Rev. Lett. 81, 4404 (1998).

[14] M. Nauroth and W. Kob, Phys. Rev. E 55, 657 (1997).

[15] E. Andrejew and J. Baschnagel, Physica A 233, 117 (1996).

[16] W. Kob, F. Sciortino and P. Tartaglia, preprint cond-mat/.

[17] J.-P. Hansen and I. R. McDonald, Theory of Simple Liquids (Academic, London, 1986).

[18] A. Latz, to be published.

[19] U. Bengtzelius, W. Götze and A. Sjölander, J. Phys. C 17, 5915 (1984).

[20] T. Gleim and W. Kob, preprint cond-mat/9902003. 
[21] S. F. Edwards and P. W. Anderson, J. Phys.: Metal Phys. 5, 965 (1975).

[22] L. Cugliandolo and P. Le Doussal, Phys. Rev. E 53, 1525 (1996).

[23] T. Franosch, M. Fuchs, W. Götze, M. R. Mayr, and A. P. Singh, Phys. Rev. E 55, 7153 (1997); M. Fuchs, W. Götze, and M. R. Mayr, Phys. Rev. E 58, 3384 (1998).

[24] G. F. Signorini, J.-L. Barrat, and M. L. Klein, J. Chem. Phys. 92, 1294 (1990).

[25] J. Horbach and W. Kob, preprint cond-mat/9901067, Phys. Rev. B (in press).

[26] J. Kisker, L. Santen, M. Schreckenberg, and H. Rieger, Phys. Rev. B 53, 6418 (1996).

[27] D. S. Fisher and D. A. Huse, Phys. Rev. Lett 56, 1601 (1986); Phys. Rev. B 38, 386 (1988).

[28] U. Müssel and H. Rieger, Phys. Rev. Lett. 81, 930 (1998).

[29] W. Kob and J.-L. Barrat, Phys. Rev. Lett. 81, 931 (1998).

[30] J.-L. Barrat, J.-N. Roux, and J.-P. Hansen, Chem. Phys. 149, 197 (1990).

[31] D. Bonn, J. Tanaka, G. Wegdam, H. Kellay and J. Meunier, Europhysics Lett., 45, 52 (1999).

[32] L. Cugliandolo and J. Kurchan, Phys. Rev. Lett. 71, 173 (1993); J. Phys. A, 27, 5749 (1994).

[33] S. Franz and H. Rieger, J. Stat. Phys. 79, 749 (1995).

[34] D. Alvarez, S. Franz, and F. Ritort, Phys. Rev. B 54, 9756 (1996).

[35] E. Marinari, G. Parisi, F. Ricci-Tersenghi, and J. J. Ruiz-Lorenzo, J. Phys. A 31, 2611 (1998).

[36] S. Franz, M. Mézard, G. Parisi, and L. Peliti, Phys. Rev. Lett., 81, 1758 (1998).

[37] K. H. Fisher and J. A. Hertz, Spin Glasses, (Cambridge University Press, 1991).

[38] A well documented review can be found in G. Parisi, F. Ricci-Tersenghi, J. Ruiz-Lorenzo, preprint cond-mat/9811374.

[39] L.F. Cugliandolo and D.S. Dean, J. Phys. A 28, 4213 (1995).

[40] A. Barrat, Phys. Rev. E 57, 3629 (1998).

[41] L. Berthier, J-L. Barrat and J. Kurchan, preprint cond-mat/9903091.

[42] L. Cugliandolo, J. Kurchan and L. Peliti, Phys. Rev. E. 55, 3898 (1997).

[43] Th. M. Nieuwenhuizen, preprint cond-mat/9811390 and references therein.

[44] S. Brawer, Relaxation in viscous liquids and glasses (American Ceramic Society, Colombus, Ohio, 1985).

[45] D. Alvarez, S. Franz, and F. Ritort, Phys. Rev. B 54, 9756 (1996).

[46] M. Mézard, G. Parisi, preprint condmat/9812180

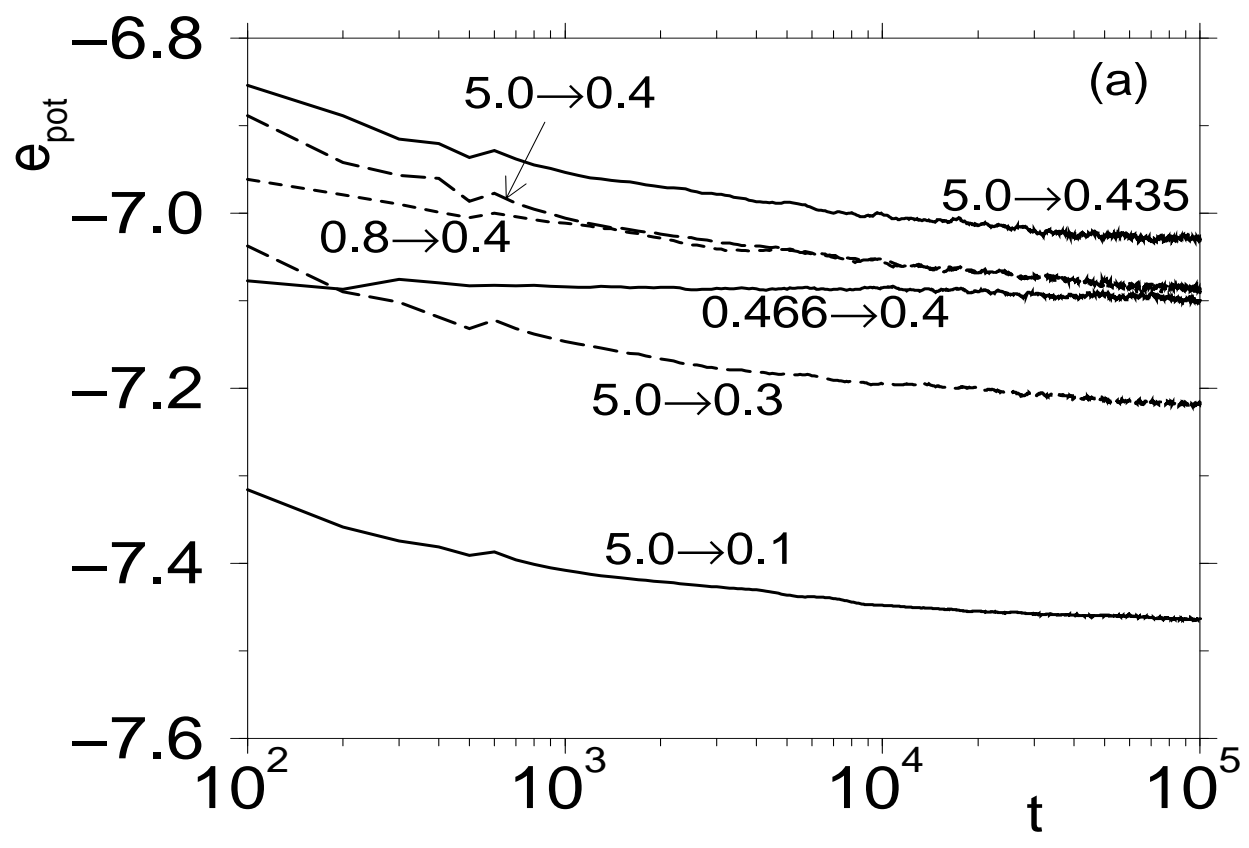




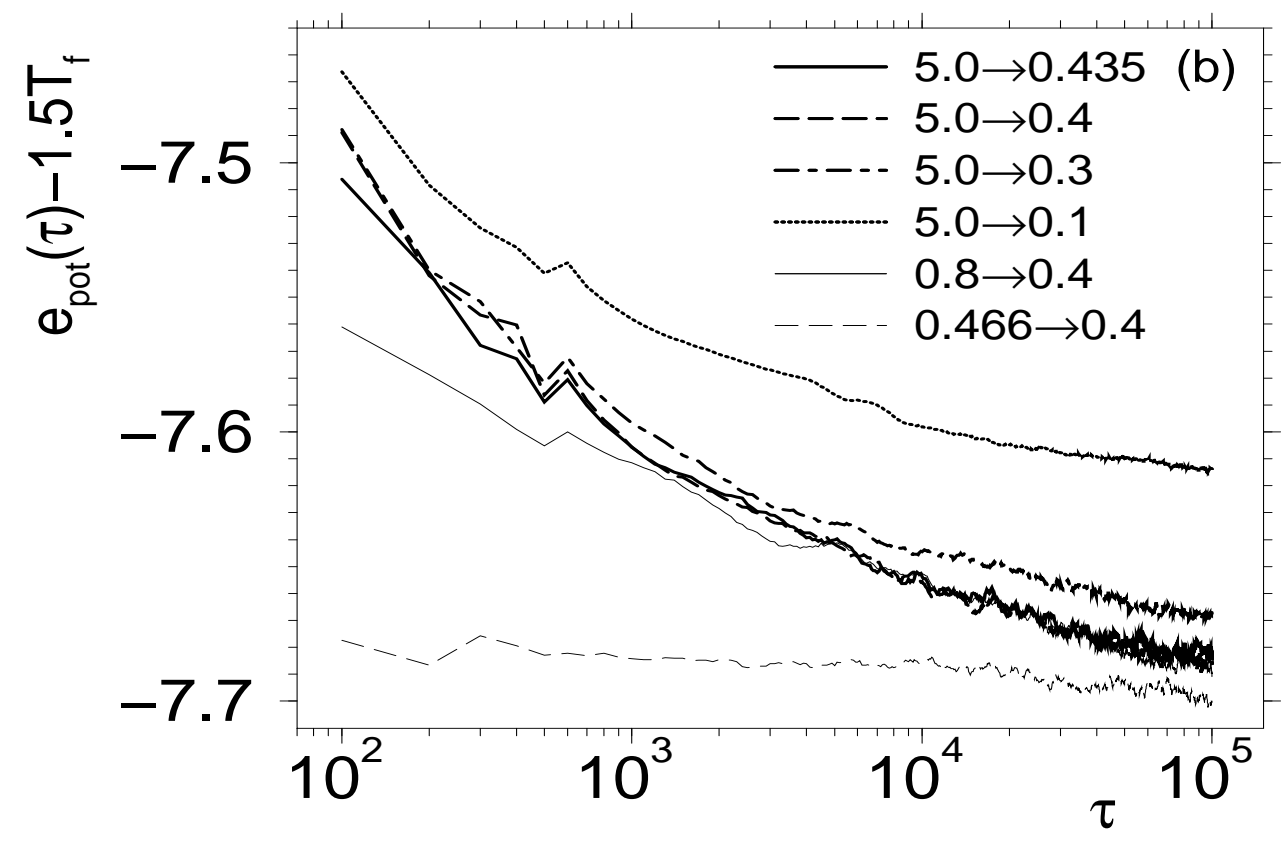

FIG. 1. a) Time dependence of the potential energy for various combinations of $T_{i}$ and $T_{f}$. b) The same date as in a) but shifted by $3 / 2 T_{f}$.

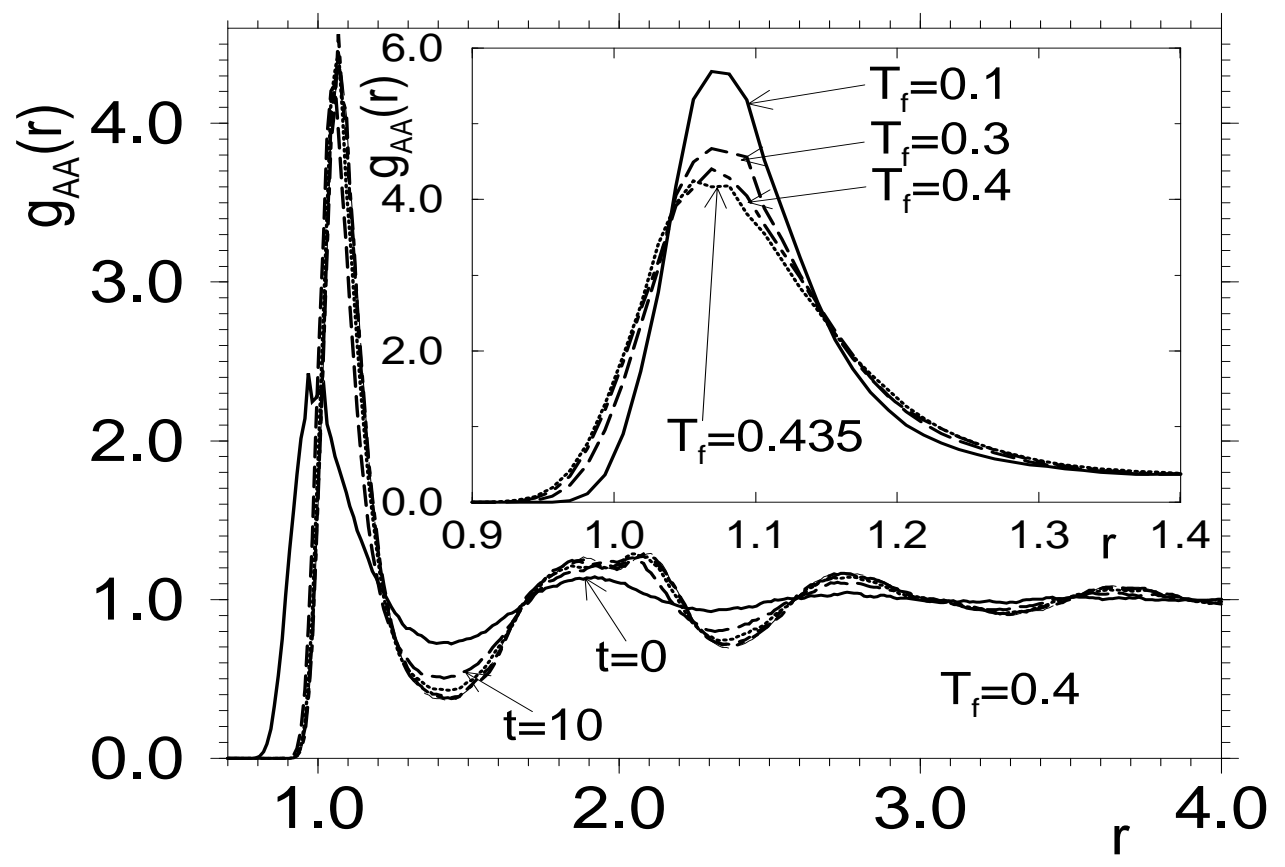

FIG. 2. Main figure: radial distribution function $g_{\mathrm{AA}}(r)$ for times $t=0$ (before the quench) and $t=10,100,1000,10000$, and 63100. $T_{i}=5.0, T_{f}=0.4$. Inset: $g_{\mathrm{AA}}(r)$ at long times for $T_{i}=5.0$ and different values of $T_{f}$. 


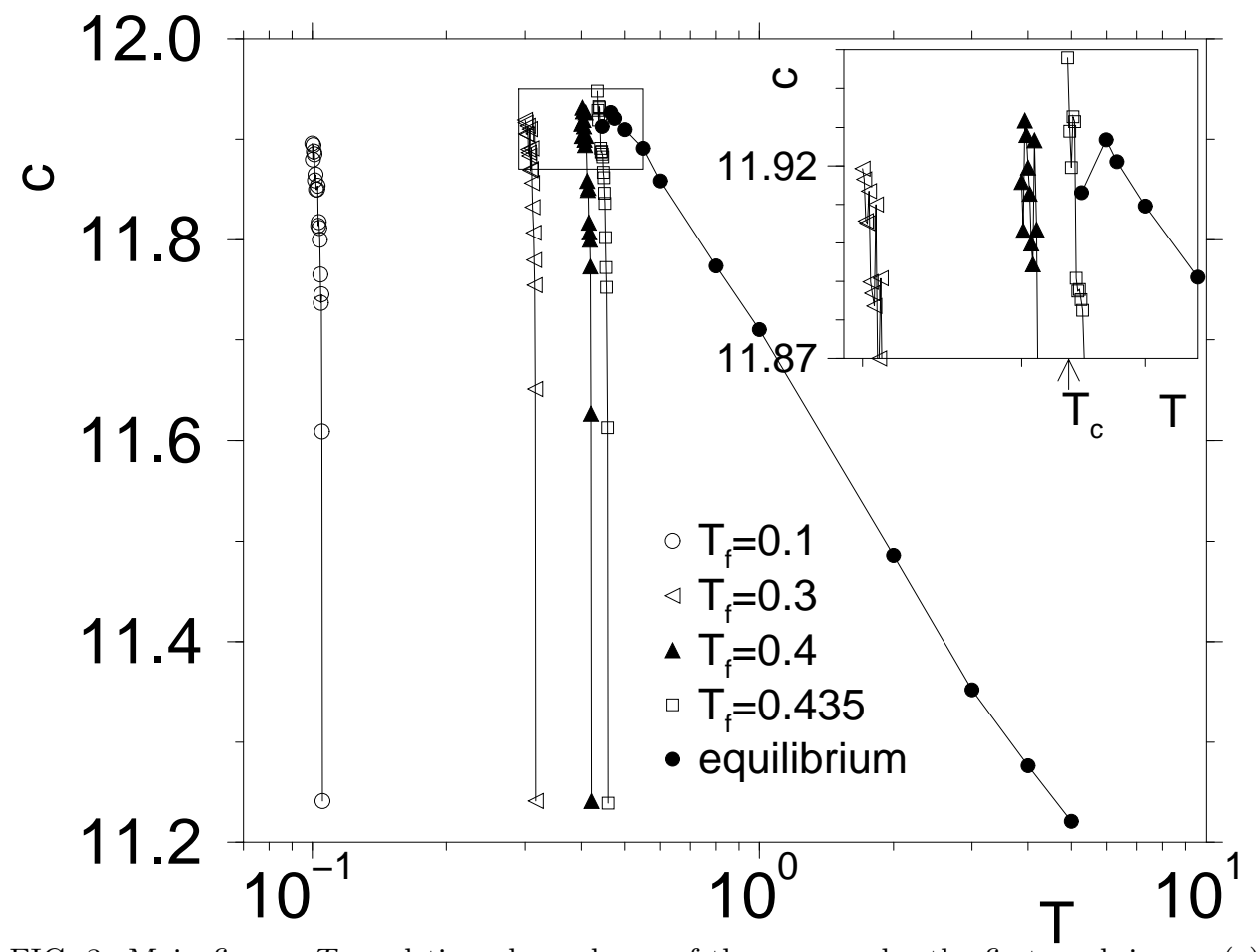

FIG. 3. Main figure: $T_{f}$ and time dependence of the area under the first peak in $g_{\mathrm{AA}}(r)$ (open symbols). Closed symbols: Temperature dependence of this quantity in equilibrium. Inset: Enlargement of the region maked by a box in the main figure.

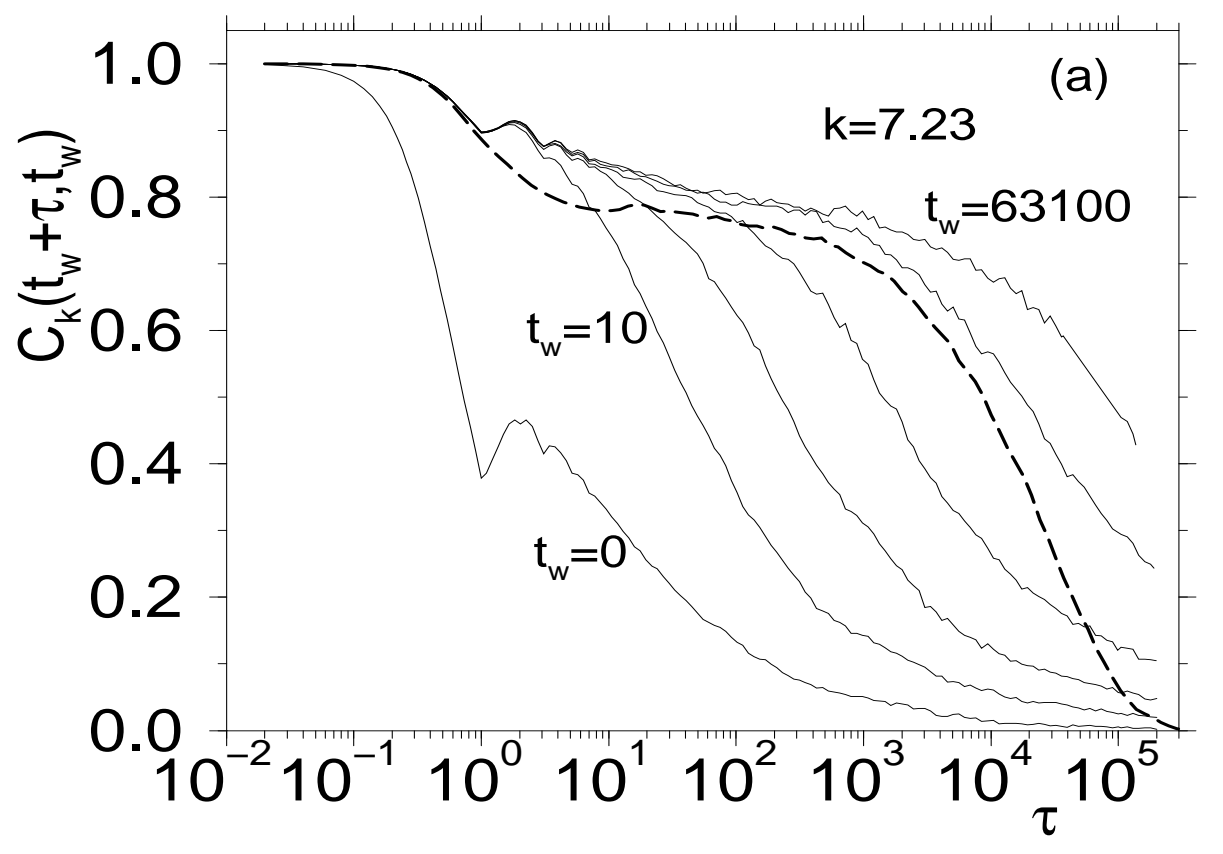




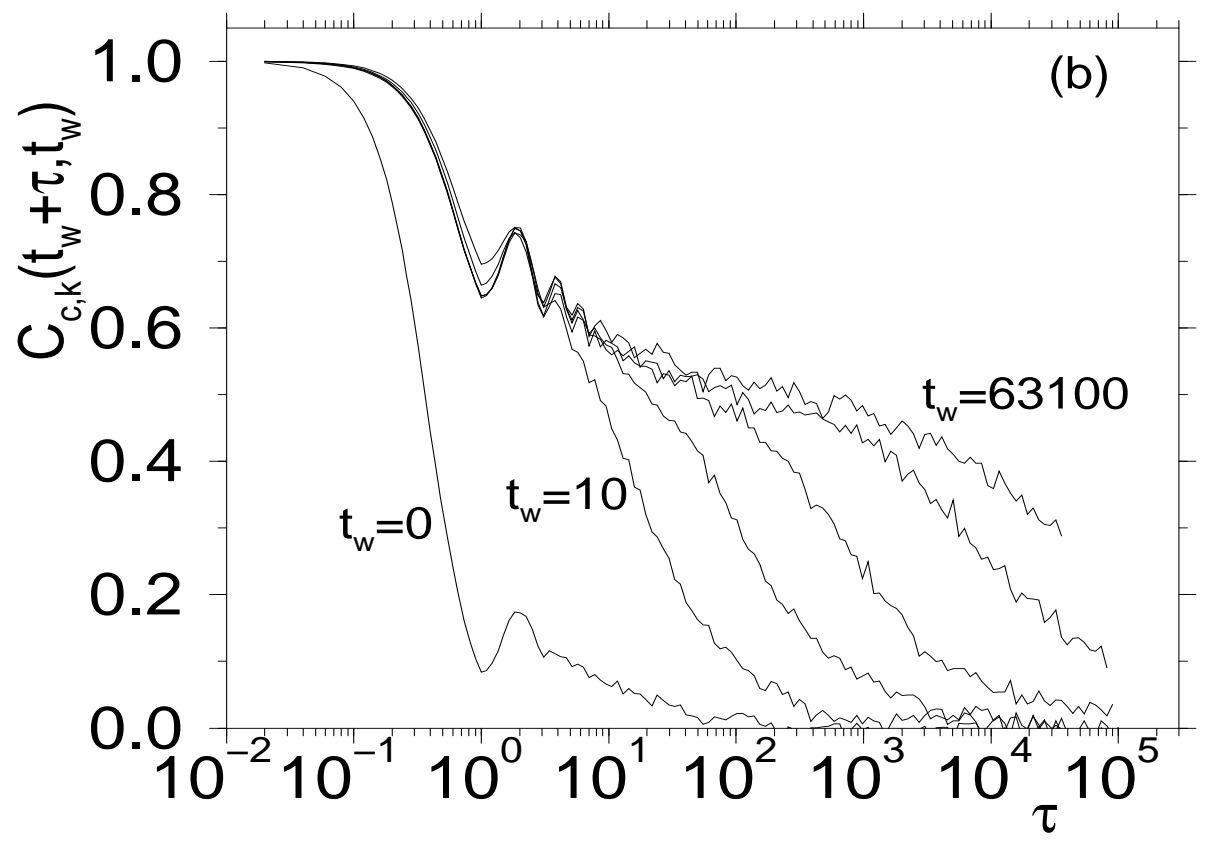

FIG. 4. Time dependence of the correlation functions $C_{k}\left(t_{w}+\tau, t_{w}\right)$, pannel (a), and $C_{c, k}\left(t_{w}+\tau, t_{w}\right)$, pannel (b), for the waiting times $t_{w}=0,10,100,1000,10000$, and 63100. In pannel (a) we have also included an equilibrium curve at higher temperature (dashed line). See text for details.

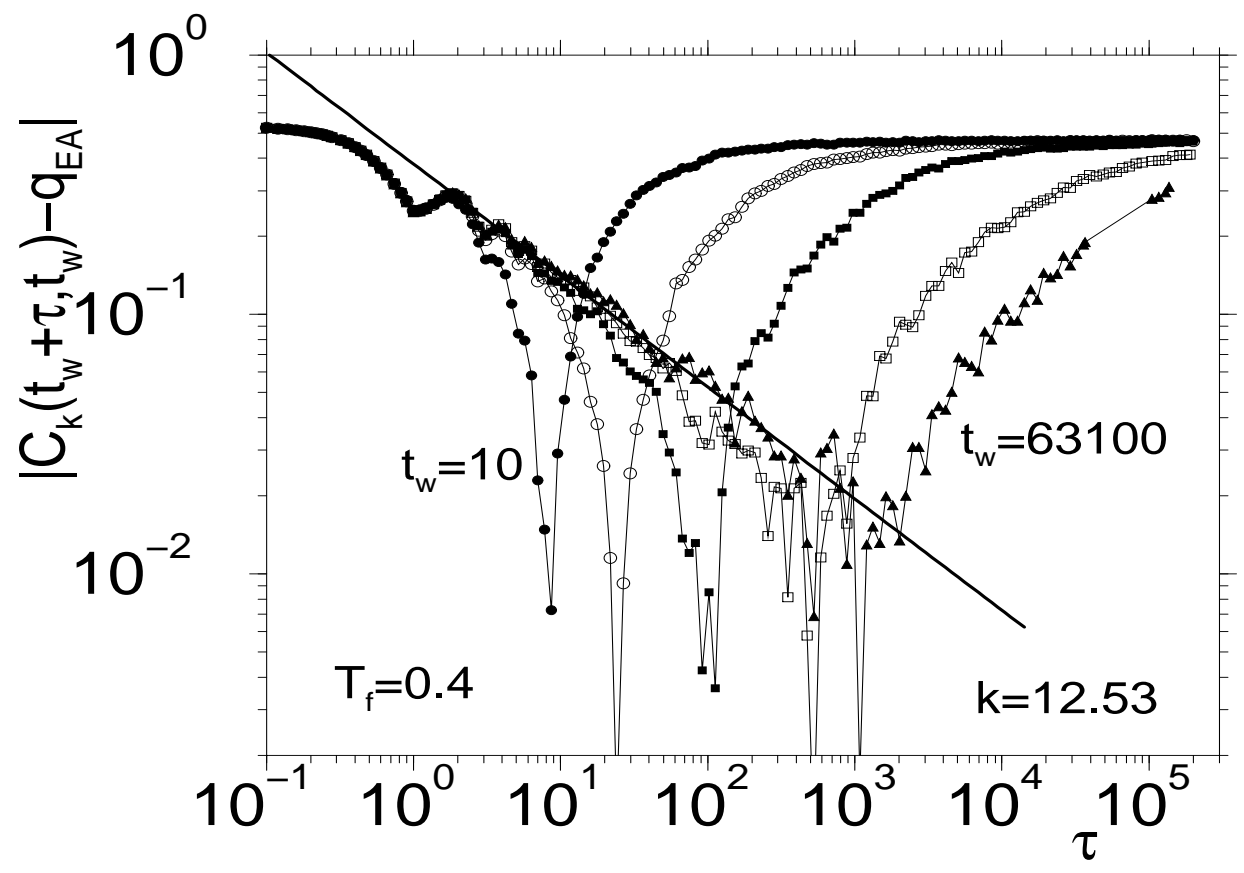

FIG. 5. Test of the presence of a power-law at short times in $C_{k}\left(t_{w}+\tau, t_{w}\right)$. The value of $q_{\mathrm{EA}}$ is $0.47 . t_{w}=10,100,1000$, 10000, and 63100. The bold straight line has slope 0.42 . 


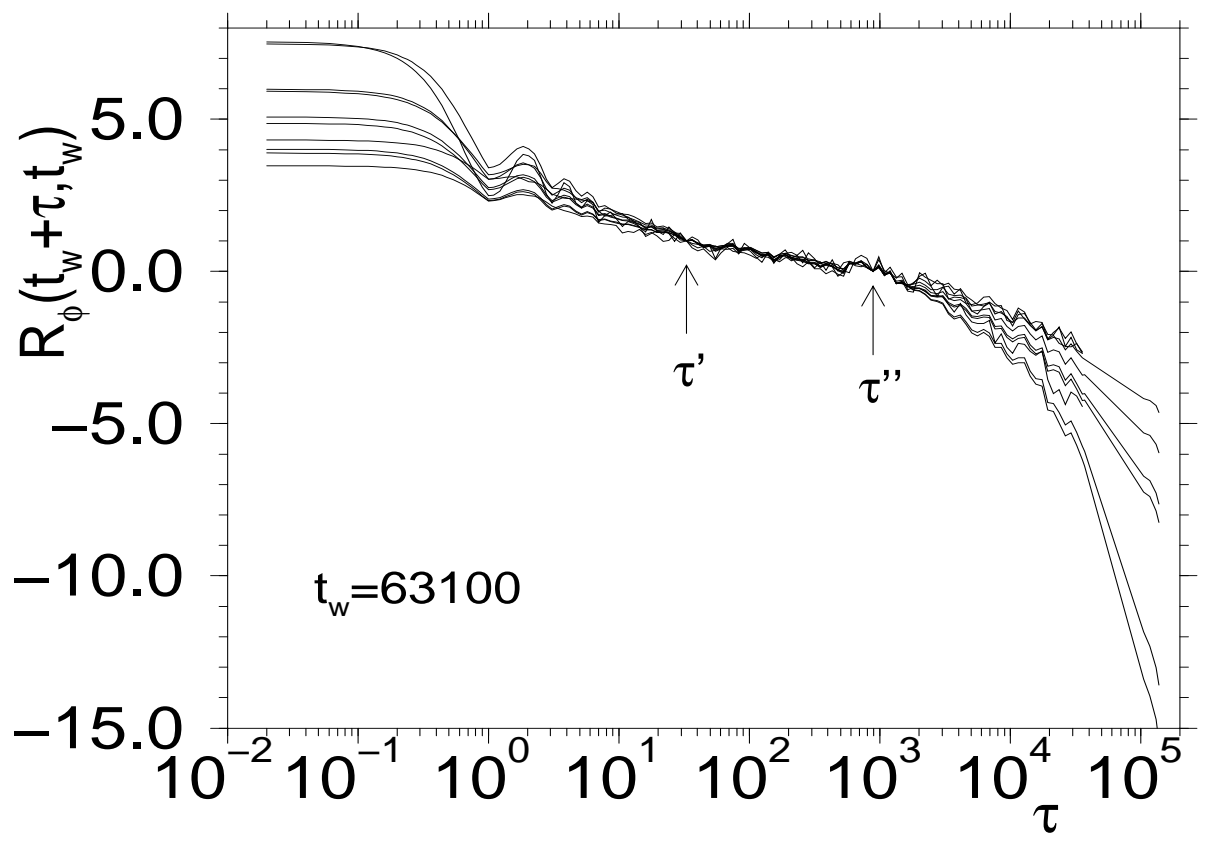

FIG. 6. Time dependence of the ratio $R_{\phi}\left(t_{w}+\tau, t_{w}\right)$ for different correlation functions (see text). The vertical arrows show the values of the times $\tau^{\prime}$ and $\tau^{\prime \prime}$ used to calculate $R_{\phi}$.

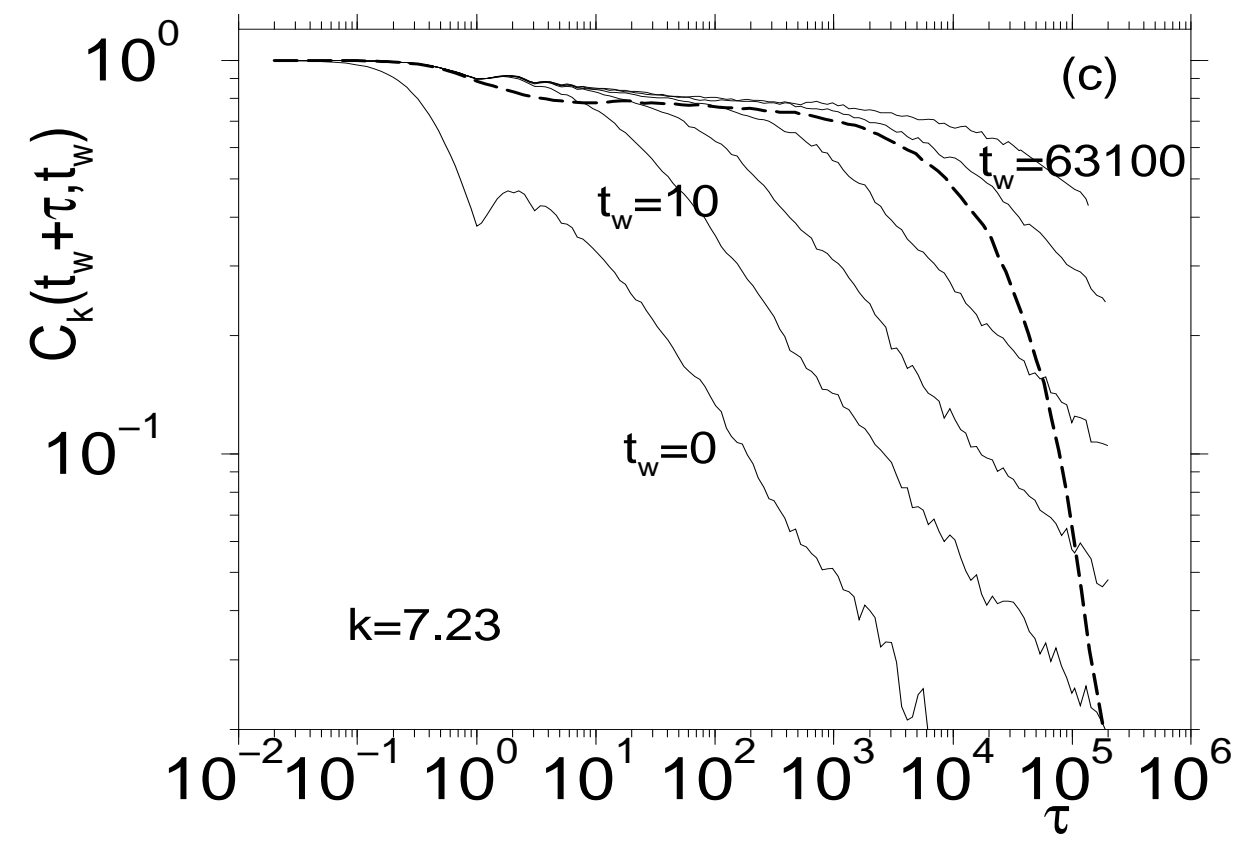




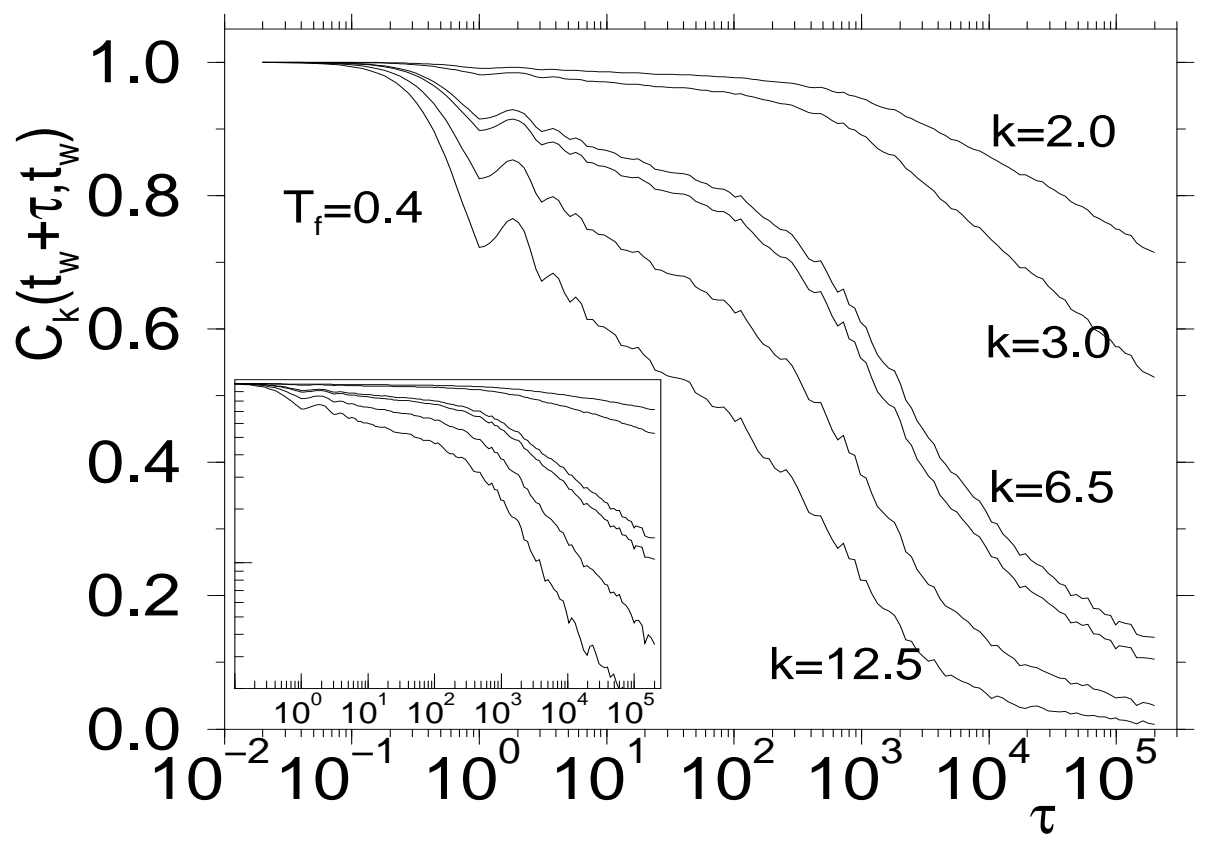

FIG. 7. a) Double logarithmic plot of $C_{k}\left(t_{w}+\tau, t_{w}\right)$ for the aging dynamics (solid lines) and the equilibrium dynamics (dashed curve). The waiting times are: $t_{w}=0,10,100,1000,10000$, and 63100. b) Wave-vector dependence of $C_{k}\left(t_{w}+\tau, t_{w}\right)$ for the waiting time $t_{w}=1000$. From top to bottom the values of $k$ are 2.0, 3.0, 6.5, 7.23, 9.6, and 12.5. Inset: the same curves in a double logarithmic presentation.

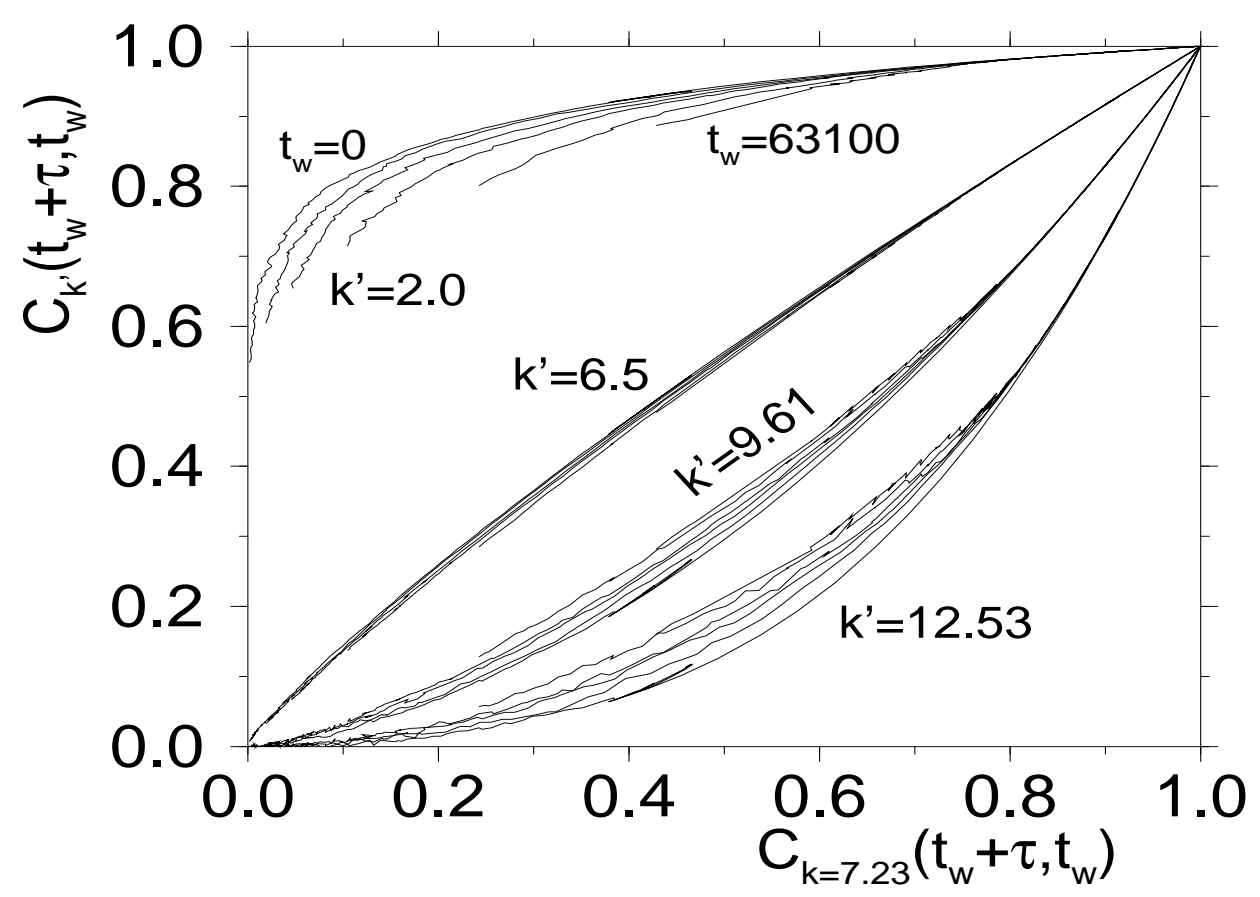

FIG. 8. Parametric plot between different correlation functions (see axis labels). The different curves for given wave-vector correspond to different values of the waiting time. $T_{f}=0.4$. 


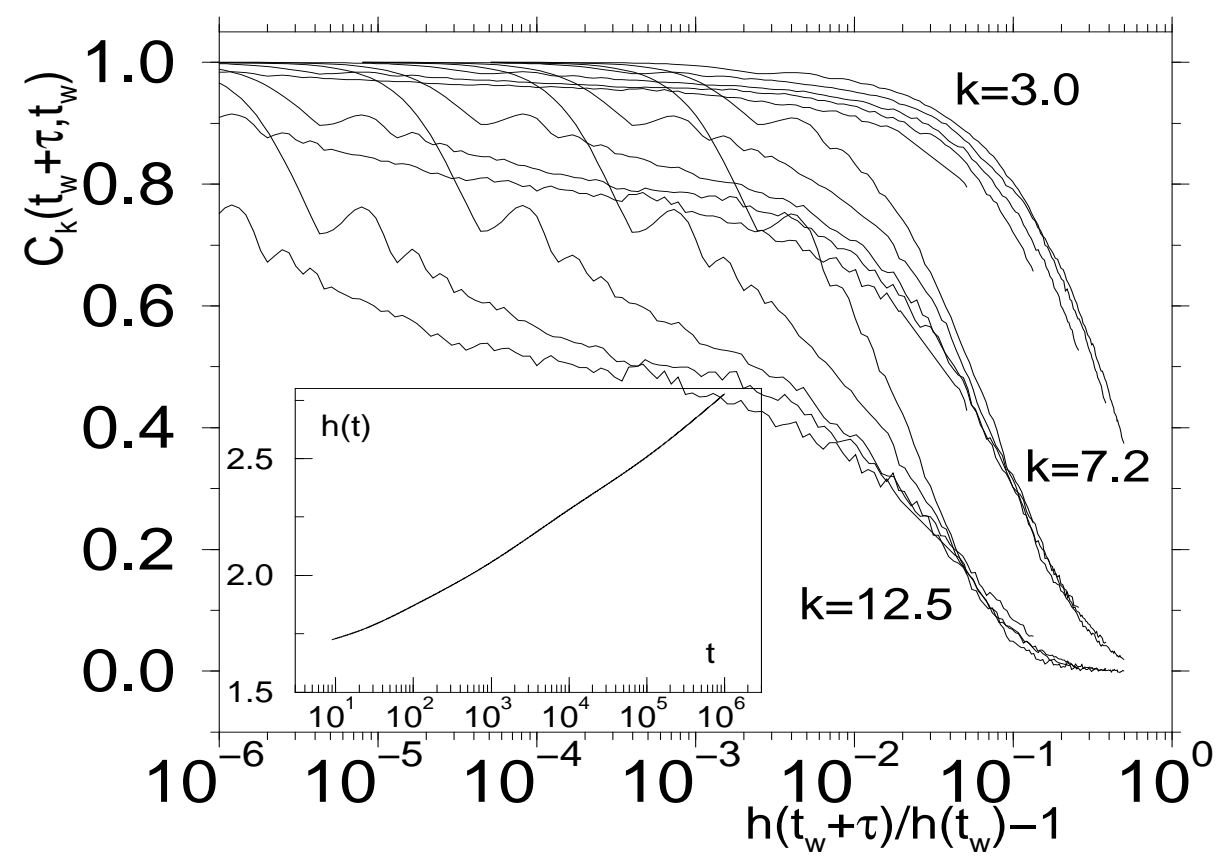

FIG. 9. Main figure: The two time correlation function as a function of the variable $h\left(\tau+t_{w}\right) / h\left(t_{w}\right)-1$ for different wave-vectors and waiting times (see text). The function $h(t)$ was chosen to make the curves for $k=7.23$ collapse at long times and its time dependence is shown in the inset.

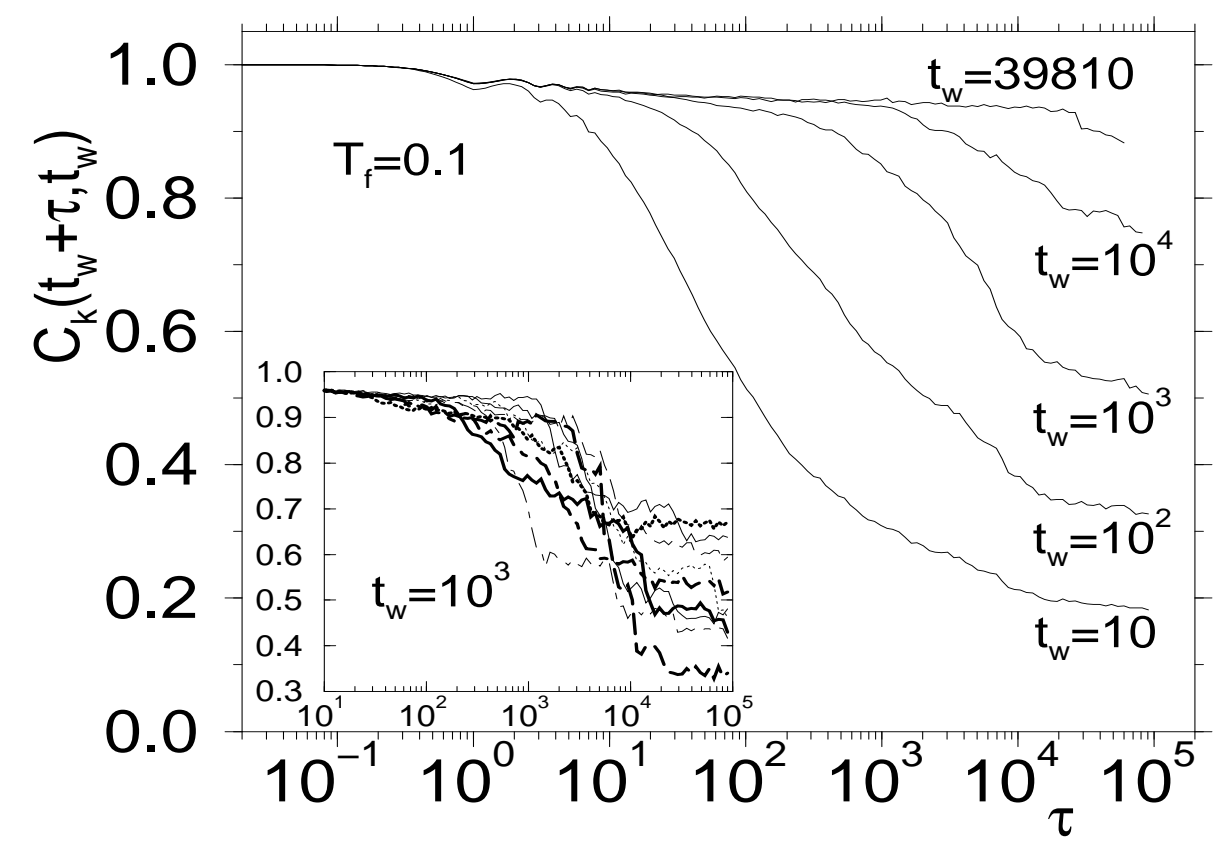

FIG. 10. Main figure: Time dependence of the correlation functions $C_{k}\left(t_{w}+\tau, t_{w}\right)$, for the waiting times $t_{w}=10,100,1000$, 10000, and 39810. $T_{f}=0.1, k=7.23$. Inset: The same correlation function for $t_{w}=1000$ for the individual runs. 


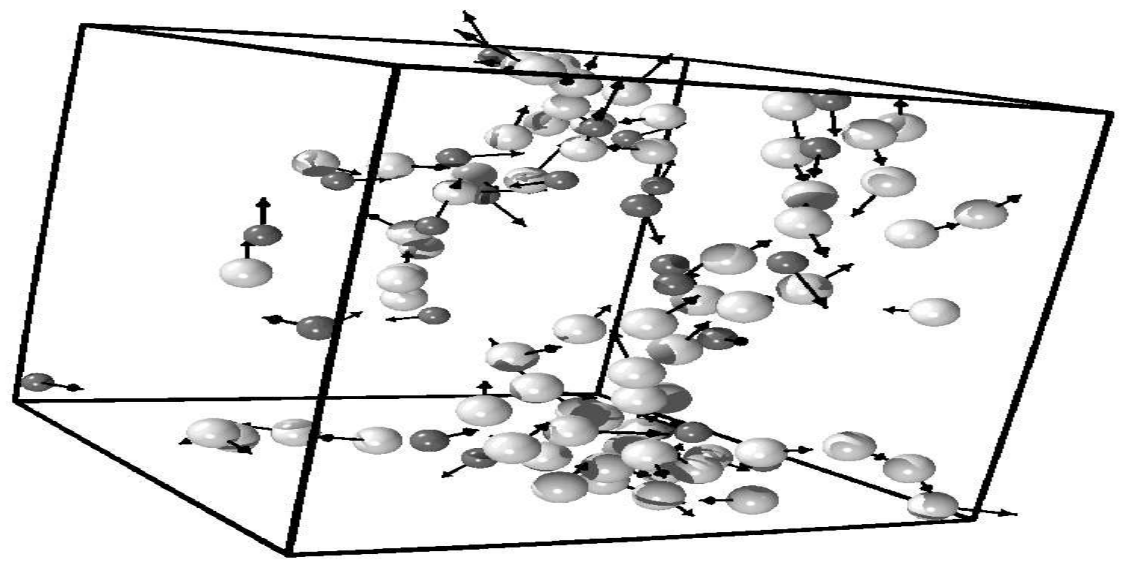

FIG. 11. Snapshot of the configuration just before $(\tau=5070$, spheres $)$ and just after $(\tau=7650$, tip of arrows $)$ the large drop in the time correlation function. In this event $C_{k}\left(t_{w}+\tau, t_{w}\right), k=7.23$, decayed from 0.79 to 0.52 . $T_{f}=0.1$.

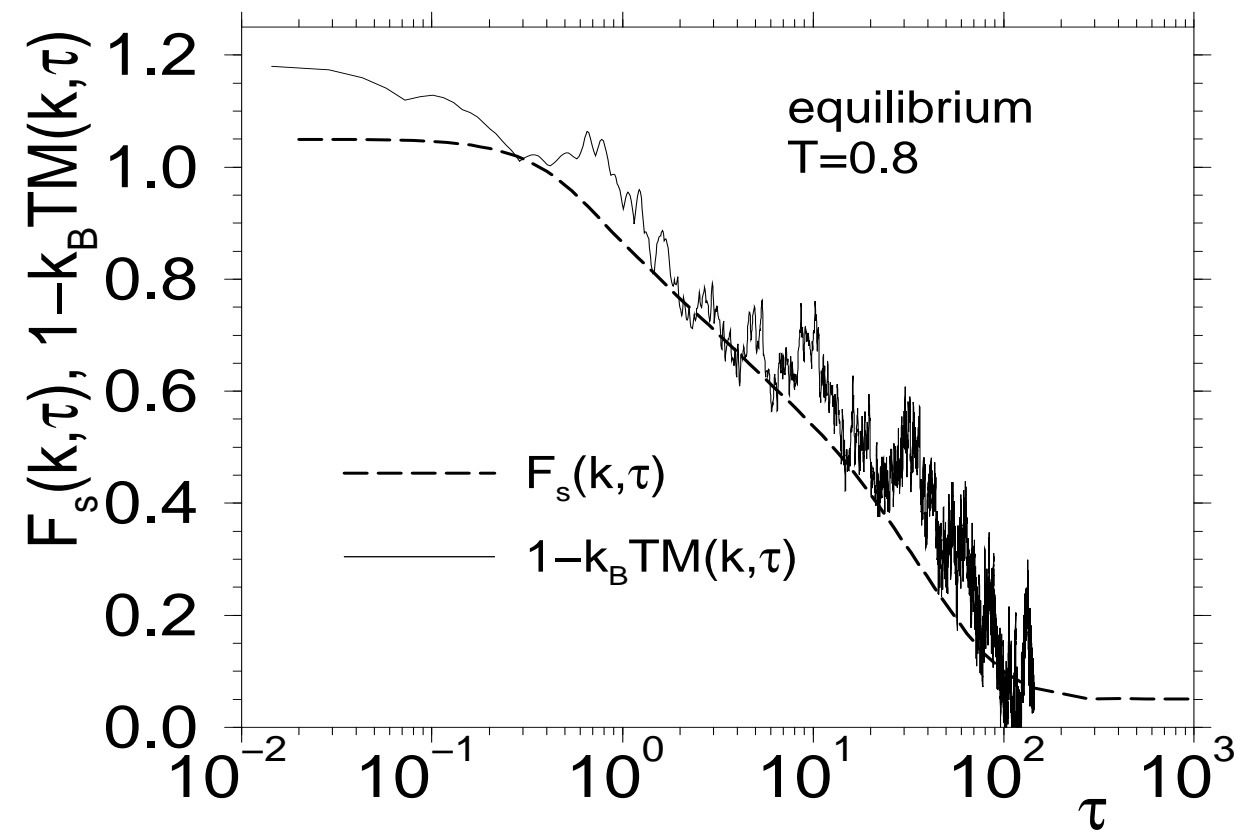

FIG. 12. Test of FDT at equilibrium. The dashed curve is the intermediate scattering function $F_{s}(k, \tau)$ for $k=7.23$ and the solid curve is the corrsponding response, i.e. $1-k_{B} T M(k, \tau)$. The response data was obtained by averaging over 14 different runs. 


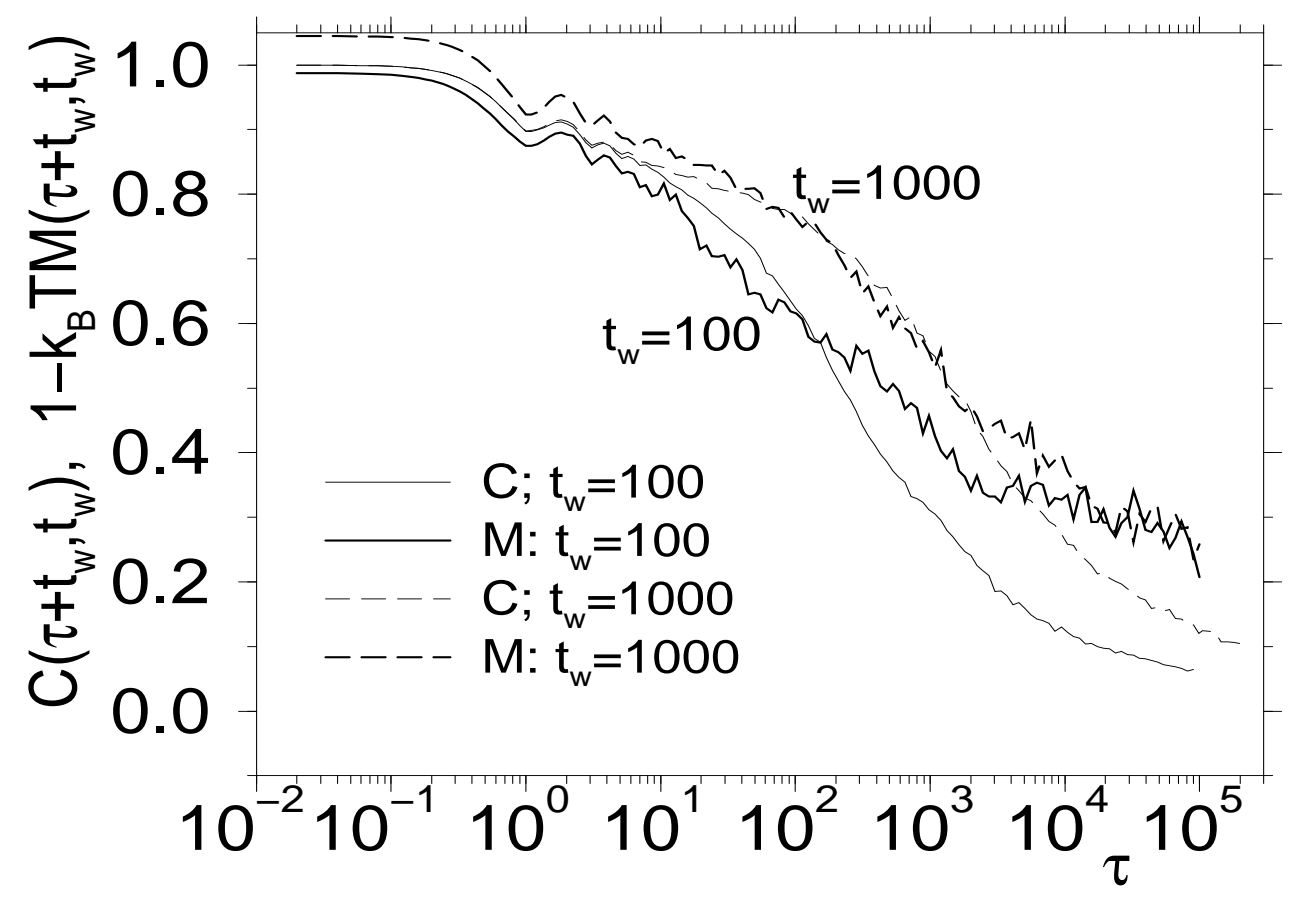

FIG. 13. Integrated response function $M$ and correlation function for the out of equilibrium case.

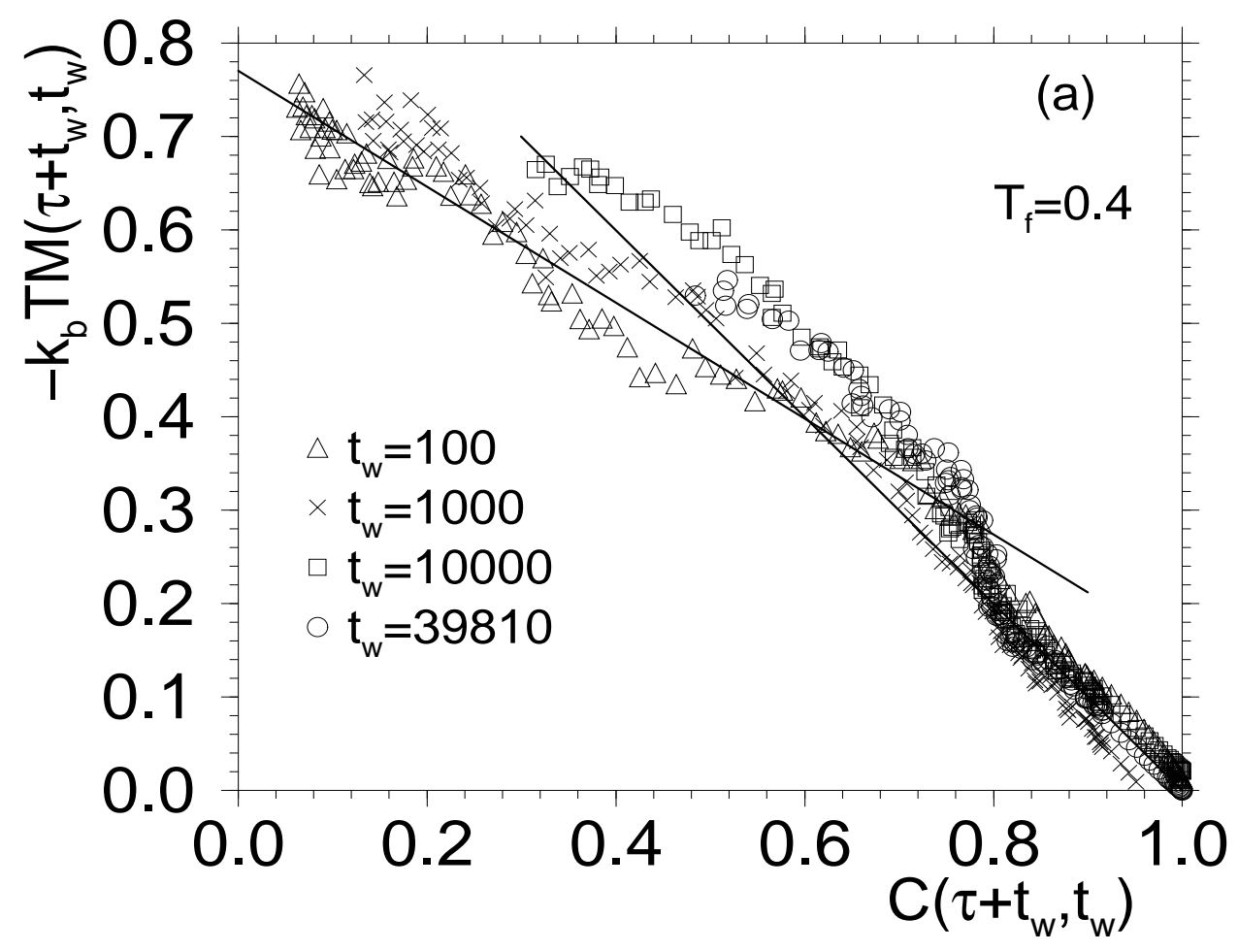



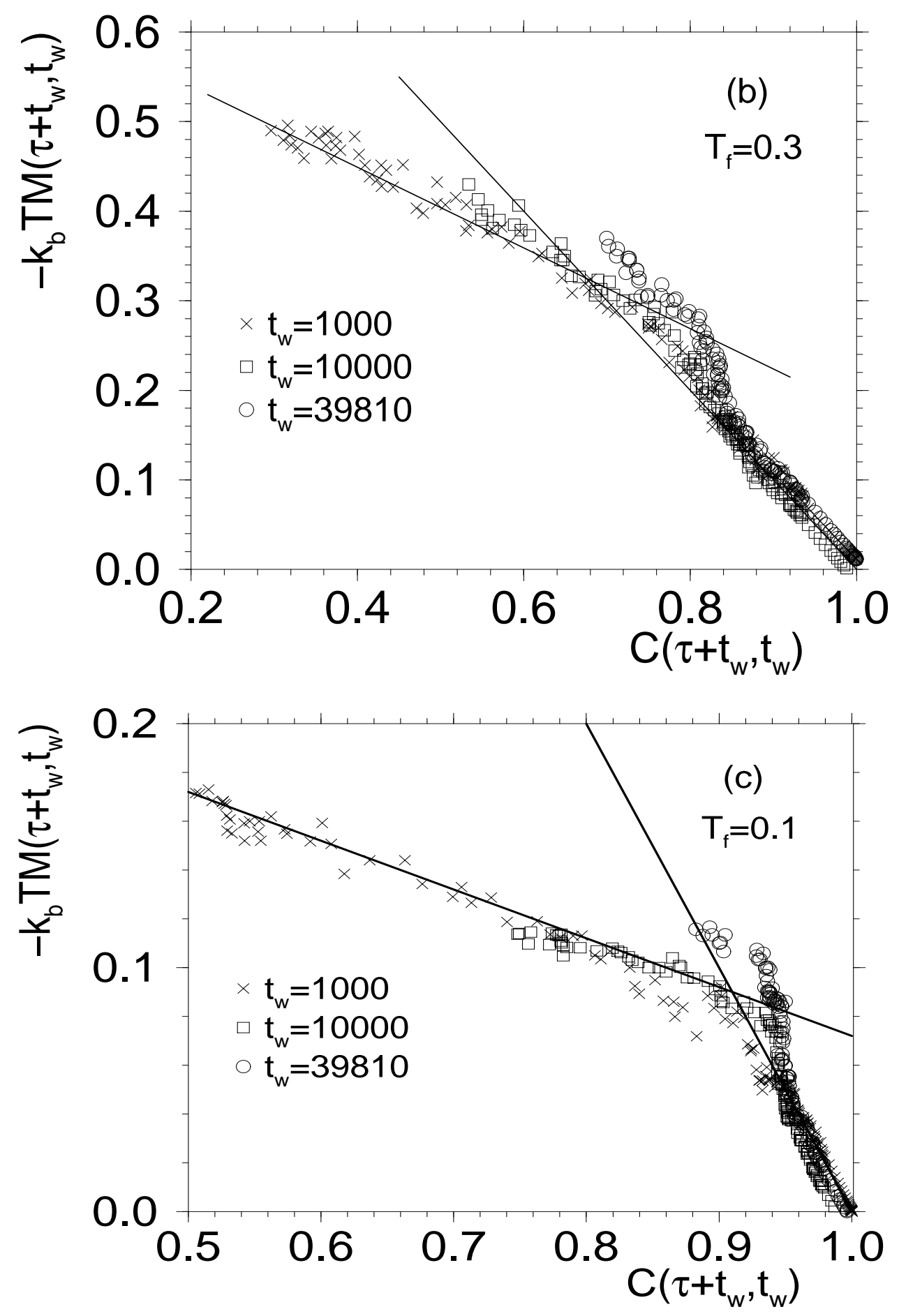

FIG. 14. Parametric plots a) $T_{f}=0.4$ b) $T_{f}=0.3$ c) $T_{f}=0.1$ 